\title{
Breaking symmetries in induced-charge electro-osmosis and electrophoresis
}

\author{
By TODD M. SQUIRES ${ }^{1} \dagger$ AND MARTIN Z. BAZANT \\ ${ }^{1}$ Departments of Applied and Computational Mathematics and Physics, California Institute \\ of Technology, Pasadena, CA 91125, USA \\ ${ }^{2}$ Department of Mathematics and Institute for Soldier Nanotechnologies, Massachusetts Institute \\ of Technology, Cambridge, MA 02139, USA
}

(Received 20 July 2005 and in revised form 30 December 2005)

Building on our recent work on induced-charge electro-osmosis (ICEO) and electrophoresis (ICEP), as well as the Russian literature on spherical metal colloids, we examine the rich consequences of broken geometric and field symmetries upon the ICEO flow around conducting bodies. Through a variety of paradigmatic examples involving ideally polarizable (e.g. metal) bodies with thin double layers in weak fields, we demonstrate that spatial asymmetry generally leads to a net pumping of fluid past the body by ICEO, or, in the case of a freely suspended colloidal particle, translation and/or rotation by ICEP. We have chosen model systems that are simple enough to admit analysis, yet which contain the most important broken symmetries. Specifically, we consider (i) symmetrically shaped bodies with inhomogeneous surface properties, (ii) 'nearly symmetric' shapes (using a boundary perturbation scheme), (iii) highly asymmetric bodies composed of two symmetric bodies tethered together, (iv) symmetric conductors in electric-field gradients, and (v) arbitrarily shaped conductors in general non-uniform fields in two dimensions (using complex analysis). In non-uniform fields, ICEO flow and ICEP motion exist in addition to the more familiar dielectrophoretic forces and torques on the bodies (which also vary with the square of the electric field). We treat all of these problems in two and three dimensions, so our study has relevence for both colloids and microfluidics. In the colloidal context, we describe principles to 'design' polarizable particles which rotate to orient themselves and translate steadily in a desired direction in a DC or AC electric field. We also describe 'ICEO spinners' that rotate continuously in AC fields of arbitrary direction, although we show that 'near spheres' with small helical perturbations do not rotate, to leading order in the shape perturbation. In the microfluidic context, strong and steady flows can be driven by small AC potentials applied to systems containing asymmetric structures, which holds promise for portable or implantable self-powered devices. These results build upon and generalize recent studies in AC electro-osmosis (ACEO). Unlike ACEO, however, the inducing surfaces in ICEO can be physically distinct from the driving electrodes, increasing the frequency range and geometries available.

\section{Introduction}

Electrokinetic phenomena involve the interaction between ionic screening clouds, applied electric fields, and low-Reynolds number hydrodynamic flows. They have

$\dagger$ Present address: Department of Chemical Engineering, University of California, Santa Barbara, CA 93106, USA. 
long played a central role in colloid and interface science (Dukhin \& Derjaguin 1974; Dukhin \& Shilov 1974; Anderson 1989; Russel, Saville \& Schowalter 1989; Lyklema 1995), analytical chemistry and separation science (Giddings 1991), and electrochemistry (Newman 1991). The basic physical mechanism is as follows. An ionic screening cloud, or double layer, forms around a charged solid surface in a liquid electrolyte. This double layer is typically thin $(\sim \mathrm{nm})$ and can often be considered small compared to other geometric features in the system (as we do here). An externally applied electric field exerts a force on the ions in the double layer, giving rise to a fluid flow that exponentially approaches the Smoluchowski 'slip velocity' just outside the charge double layer,

$$
u_{s}=-\frac{\varepsilon \zeta}{\eta} E_{\|} .
$$

Here $\varepsilon$ and $\eta$ are the dielectric constant and viscosity of the liquid (typically water), $E_{\|}$is the component of the applied electric field tangent to the surface, and $\zeta$ is the 'zeta potential', or the potential drop across the screening cloud. When the surface is held fixed, $u_{s}$ drives a flow termed electro-osmosis; when the surface defines a freely suspended particle, the particle moves via electrophoresis.

Recent years have seen a tremendous effort towards developing microfluidic 'labs on a chip' for miniaturized, automated and parallelized experiments (see, e.g. Reyes et al. (2002) for a historical review). Electrokinetics plays the key role in many microfluidic separation and analysis devices (Bruin 2000; Verpoorte 2002; Lion et al. 2003; Tegenfeldt et al. 2004; Ugaz et al. 2004), and interest in this classic subject has thus been renewed (Viovy 2000; Slater et al. 2003; Stone, Stroock \& Ajdari 2004; Squires \& Quake 2005). In most cases, electrophoresis is used for separations. Electro-osmosis has also been explored as a fluidic manipulation tool, although various disadvantages (discussed below) preclude its widespread use in actual devices.

\section{1. 'Induced-charge' electrokinetic phenomena}

This is the second in a pair of in-depth papers on induced-charge electro-osmosis (ICEO) at polarizable (metallic or dielectric) surfaces, whose basic ideas we have summarized in the first paper in the context of microfluidic applications (Bazant \& Squires 2004). Our original motivation was to identify the essential physics behind 'AC electro-osmosis' at micro-electrode arrays, discovered independently by Ramos et al. (1999) in experiments and by Ajdari (2000) in theoretical calculations. We showed that the basic slip mechanism, which we call 'ICEO', requires neither electrodes nor $\mathrm{AC}$ voltages and can arise in many other contexts. For example, we gave some new microfluidic examples of ICEO flows around dielectrics and conductors of either fixed total charge or fixed potential in general DC or AC applied fields, which have since been observed in experiments by Levitan et al. (2005).

We also pointed out that similar flows had been studied in the Russian literature since the 1980s in the seemingly different context of metal colloids (Murtsovkin 1996), although this imporant work had gained little (if any) international attention. In particular, the ICEO flow around an uncharged metal sphere was first predicted by Gamayunov, Murtsovkin \& Dukhin (1986) and later observed, at least qualitatively, in a few experiments (see below). Earlier still, the electrophoresis of a charged metal sphere had also been considered in the school of Dukhin \& Derjaguin (1974) occasionally since (at least) the time of Levich (1962), although the electrophoretic mobility (which is unaffected by ICEO) was emphasized, rather than the (strongly influenced) flow profile. In general, more attention was given to the induced dipole 
(a)

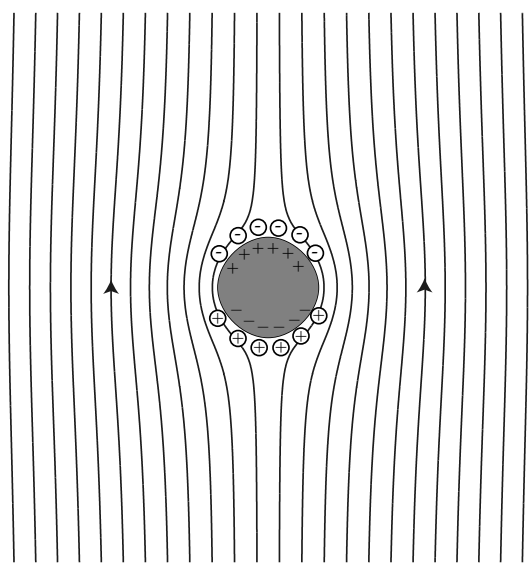

(b)

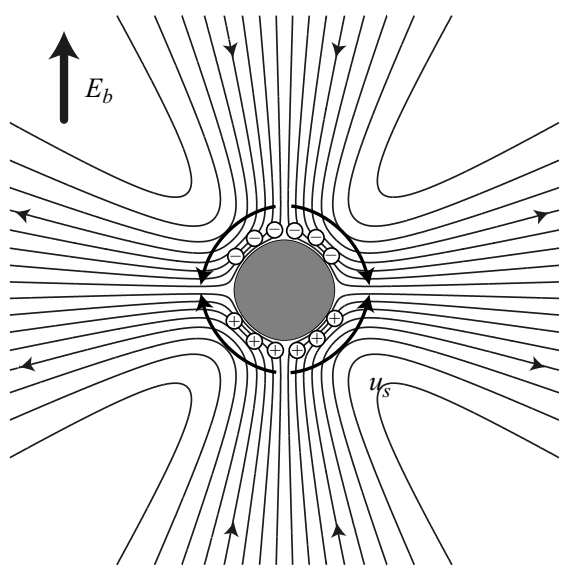

FIGURE 1. A representation of induced-charge electro-osmotic (ICEO) flow: $(a)$ in steady-state, an induced charge cloud, dipolar in nature, is established in order that no field line (and therefore electrolytic current) terminates at the surface of the conducting body. (b) The steady-state electric field drives the dipolar induced charge cloud, setting up a quadrupolar ICEO flow.

moment and its effect on dielectrophoresis rather than the associated electrokinetic flows in polarizable colloids (Dukhin \& Shilov 1974, 1980). All of these studies fit into the larger context of 'non-equilibrium electro-surface phenomena' in colloids, studied extensively in the Soviet Union since the 1960s (Dukhin 1993). This work deserves renewed attention from the perspective of designing colloids and microfluidic devices, since geometrical complexity (the focus of this paper) can now be engineered to control flows and particle motions, in ways not anticipated by the many earlier studies of ideal colloidal spheres.

Let us briefly review how ICEO differs from standard, 'fixed-charge' electro-osmosis. Both effects involve an electro-osmotic flow that occurs because of the action of an applied field upon the diffuse cloud of screening ions that accumulates near a surface. The key difference between standard electro-osmosis and ICEO lies in the nature of the screening cloud itself (and thus $\zeta$ ). In standard electro-osmosis, the zeta potential is an equilibrium material property of the surface and is thus typically taken to be constant. In contrast, ICEO flows (around conducting or polarizable surfaces) involve a charge cloud that is induced by the applied field itself, giving a non-uniform induced zeta potential of magnitude $E_{b} a$, where $a$ is a geometric length scale characteristic of the body. The velocity scale for ICEO,

$$
U_{0}=\frac{\varepsilon a E_{b}^{2}}{\eta},
$$

depends on the square of the electric field, so a non-zero average ICEO flow persists even in an AC electric field.

Figure 1 illustrates the phenomenon. Consider an inert (ideally polarizable) conducting body immersed in an electrolyte subject to a suddenly applied electric field, so the electric field lines initially intersect the conducting surface at right angles in order to satisfy the equipotential boundary condition. The electric field drives an ionic current in the electrolyte, however, and ions cannot penetrate the solid/liquid surface without electrochemical reactions. Instead, at low enough voltages to ignore surface conduction (see below), the ions that intersect the conducting surface are stopped 
and accumulate in the double layer. This induced charge cloud grows and expels field lines, until none intersect the conducting surface, as shown in figure 1(a). The induced charge cloud is dipolar in character, giving a quadrupolar ICEO flow, as seen in figure $1(b)$. Similar, only weaker, ICEO flows occur around dielectrics, but for simplicity here we will focus on ideally polarizable conducting bodies.

The dynamics of double-layer charging at an electrode (or more generally, a polarizable surface) is a subtle problem with a long and colourful history (Bazant, Thornton \& Ajdari 2004). In the 'weakly nonlinear' regime where we perform our analysis, perturbations to the bulk ionic concentrations are negligible beyond the Debye (or Gouy) screening length $\lambda_{D}$, assumed to be much smaller than the geometrical scale, $\lambda_{D} \ll a$. In this limit, the electric field is determined, independent of any fluid flow, by an equivalent circuit model consisting of a homogeneous bulk resistor coupled to double-layer capacitors. The ' $\mathrm{RC}$ time' for charging these capacitors and screening the bulk field thus involves a product of the two length scales,

$$
\tau_{c}=\frac{\lambda_{D} a}{D},
$$

where $D$ is a characteristic ionic diffusivity.

In the present context, this classical circuit model has been applied to metal colloidal spheres (Simonov \& Shilov 1977; Squires \& Bazant 2004) and linear micro-electrode arrays (Ramos et al. 1999; Ajdari 2000; Gonzalez et al. 2000), where $\tau_{c}^{-1}$ appears as the critical frequency for AC electro-osmosis. In less simple situations, such as many given below, more than one length scale characterizes the geometry, and thus the frequency response can be complicated. Nevertheless, the longest length scale is still associated with the longest time scale via (1.3), as long as the voltage is small enough not to perturb the bulk concentration (which would introduce the longer time scale for bulk diffusion, $\left.a^{2} / D\right)$. Since our goal here is to expose the rich spatial dependence of ICEO flows, we postpone a careful study of their time dependence for future work; thus we consider only steady DC fields and flows, which also approximate the timeaveraged flows that occur under low-frequency AC fields $\left(\omega \ll 2 \pi / \tau_{c}\right)$.

\subsection{Breaking symmetries in electrokinetics}

In Squires \& Bazant (2004), we focused on ICEO as a means to manipulate fluids in microfluidic devices, exemplified by flows around polarizable cylindrical posts in uniform applied fields, similar to those visualized in the subsequent experiments of Levitan et al. (2005). Analogously, the Russian literature on what we call 'ICEO' in polarizable colloids has also focused on the simplest case of metal spheres (Gamayunov et al. 1986; Murtsovkin 1996), albeit with more difficult experimental verification. The theme of simple geometries also characterizes the early work on AC electro-osmosis at a symmetric pair of micro-electrodes (Ramos et al. 1999; Gonzalez et al. 2000).

In the present paper, we focus more generally on ICEO flows with broken symmetries, inspired by the work of Ajdari, who has long emphasized and explored the rich effects of asymmetry in electrokinetics, both linear (Ajdari 1995, 1996, 2002b; Long \& Ajdari 1998; Gitlin et al. 2003) and nonlinear (Ajdari 2000, 2002a; Studer et al. 2002, 2004). In the specific context of ICEO, Ajdari (2000) first predicted that an asymmetric array of electrodes, subject to $\mathrm{AC}$ forcing at a particular frequency, could function as a microfluidic pump, as Brown, Smith \& Rennie (2001), Studer et al. (2002) and Mpholo, Smith \& Brown (2003) later demonstrated experimentally, although the simple theory clearly breaks down at large voltages and large electrolyte 
concentrations (Studer et al. 2004). Ramos et al. (2003) have also begun to extend their studies of AC electro-osmosis to asymmetric pairs of electrodes, which can drive a directed fluid flow, unlike the symmetric pair of Ramos et al. (1999).

In the Russian colloids literature (Dukhin \& Shilov 1974, 1980), the induced dipole moment has been calculated for dielectric spheroids in electrolytes, but it seems there has been no theoretical work on ICEO flows around asymmetric polarizable particles, although non-uniform electric fields applied to spherical particles have been considered. Shilov \& Éstrela-Lópis (1975) were apparently the first to note that electro-osmotic flows play a major role in the classical problem of dielectrophoresis (DEP; Pohl 1978) when the fluid is an electrolyte (or 'dipolophoresis', as it was called in the Russian literature). The theory for dielectric and conducting spheres in a uniform-gradient field, including some effects of concentration polarization and surface conductance, has been developed by Shilov \& Simonova (1981) for thin double layers and by Simonova, Shilov \& Shramko (2001) for arbitrary double-layer thickness. For conductors, the effects of DEP and ICEP are in close competition, and for a metal sphere they precisely cancel to yield zero particle velocity (although not zero flow). As we show below, however, this is a very special case, since a metal cylinder (or any other shape) will generally move if free to do so, or pump fluid if it is fixed.

Otherwise, in the Russian literature, there have been a few qualitative experiments on ICEP for asymmetric (or 'anisometric') particles, and it is generally observed that nearly spherical metal particles move in AC fields, contrary to the theory for an ideal sphere. Gamayunov \& Murtsovkin (1987) and Murtsovkin \& Mantrov (1990) reported the motion of quartz particles in all possible directions in a uniform AC field, each moving toward its most pointed end. However, they could only observe particles near the walls of the experimental container, and could not say whether the resulting motion arose due to the influence of the walls, or whether this motion would also occur in the bulk of the fluid. Below we show that ICEP can drive motion toward either the blunt end or the pointed end of an arrow-like particle, depending on its precise shape, and we give simple criteria to determine the direction in which an asymmetric particle will move.

\subsection{Overview of the present work}

By departing from electrodes, AC forcing and simple geometries, our theoretical work offers many new opportunites to exploit broken symmetries to shape microfluidic flows by ICEO or to manipulate colloidal particles by ICEP, as summarized by Bazant \& Squires (2004). Building on our paper, Yariv (2005) has described general tensor relations for the translation and rotation of three-dimensional conducting particles by ICEP; he has also used the reciprocal theorem for Stokes flows (Brenner 1964; Stone \& Samuel 1996) to calculate ICEP velocities, as we do below (in both two and three dimensions). Here instead, we present detailed calculations for a variety of paradigmatic problems, which serve to demonstrate basic physical principles, and to guide the engineering design of polarizable colloids and microfluidic structures. We examine broken symmetries of the 'working' conductor as well as in the applied background field and demonstrate that ICEO fluid pumping (or ICEP motion) can occur in any direction relative to the background field by a suitable breaking of spatial symmetry.

In the microfluidic context, a notable effect of broken symmetries is to allow steady ICEO flows to be driven perpendicular to an AC applied field. In the context of linear electrokinetics, Ajdari (1996, 2002b) predicted that charge-patterned surfaces with special geometrical features can generate flows transverse to the applied field, 
which Gitlin et al. (2003) demonstrated experimentally. Such a strategy allows a strong electric field to be established by applying a relatively small potential across a microchannel. This significantly reduces the voltage required to drive a given flow over conventional electro-osmotic flow devices, and thus represents a promising candidate for portable, self-powered microfluidic devices. However, standard (linear) electroosmosis requires a steady DC voltage, which in turn necessitates electrochemical reactions that introduce bubbles or otherwise foul the fluid. Such concerns are typically addressed by placing electrodes far from the working fluid to avoid contamination, but this contradicts the advantages of transverse electro-osmosis. On the other hand, the transverse ICEO flows described here are driven by AC voltages that alleviate these problems, and may enable fully miniaturized microfluidic systems.

The ability to drive strong flows by applying small potentials across closely spaced electrodes has been a central motivation for AC electro-osmotic pumps (Ajdari 2000; Brown et al. 2001; Studer et al. 2002, 2004; Mpholo et al. 2003; Ramos et al. 2003). In such systems, the surfaces over which charge clouds are induced are the same as those to which the driving potentials are applied, giving an 'optimal' frequency for pumping and vanishing response at both low and high frequencies. By contrast, the inducing surfaces and driving electrodes in ICEO/ICEP systems can be physically distinct, increasing the range of driving frequencies available (e.g. ICEO flows persist down to zero frequency), and also allowing a broader class of geometries to be employed.

In the colloidal context, particles generally experience no net electrophoretic motion under AC applied fields, owing to the linearity of electrophoresis. The induced-charge electro-osmotic flow around asymmetric conducting or polarizable particles, however, can lead to a net particle motion with components either along or transverse to the applied field, which we call 'ICEP'. In fact, we provide simple principles to design particles which align themselves and then move either along or transverse to the applied field or which spin continuously in place at a given orientation relative to the field. Furthermore, ICEO flow is longer-ranged $\left(u \sim r^{-2}\right)$ than standard linear electrophoresis $\left(u \sim r^{-3}\right)$, and affects particle interactions in dense colloids. Indeed, this was the original motivation for studying 'ICEO flows' in the Russian literaure (Gamayunov et al. 1986).

The paper is organized as follows. Section 2 presents the mathematical model for ICEO in weak fields with thin double layers and also describes the fundamentals of dielectrophoresis. We then discuss the ICEO and ICEP of conducting bodies whose symmetry is broken owing to: a spatially asymmetric surface coating ( 3 ); a small shape asymmetry that is treated perturbatively $(\S 4)$; and a composite body composed of two electrically connected spheres or cylinders of different radii $(\S 5)$. Section 6 briefly discusses ICEO and ICEP of symmetric conducting bodies in a uniformgradient field. Finally, in $\$ 7$ the general problem of ICEO flow and multipolar DEP force is solved in two dimensions using complex analysis, for any shape in an arbitrary divergence-free background field.

\section{Basic theory for thin double layers and weak electric fields}

\subsection{Bulk electric field and induced zeta potential}

The general problem of ICEO flow around an asymmetric metal or dielectric object is complicated, so we restrict our analysis to the case of an ideally polarizable conducting body with a thin double layer, $\lambda \ll a$, in a weak electric field, $E_{b} a \ll k T / e$, as in our previous work (Squires \& Bazant 2004). We will also assume the equilibrium zeta potential to be weak, as discussed below. In this limit, the induced zeta potential 
arises from the equivalent circuit of an Ohmic bulk resistor coupled to a doublelayer capacitor on the surface, which drives a Stokes flow via surface slip given by equation (1.1). This standard circuit model for nonlinear electrokinetics (e.g. used by Gamayunov et al. 1986; Ramos et al. 1999; Ajdari 2000; Bazant \& Squires 2004) can be derived systematically by matched asymptotic expansions (Gonzalez et al. 2000; Squires \& Bazant 2004).

The circuit approximation is also valid (with a nonlinear differential capacitance) at somewhat larger applied fields, as long as the (steady-state) Dukhin number (Lyklema 1995), or its generalization for time-dependent problems (Bazant et al. 2004), remains small. The Dukhin numbert, $D u=\sigma_{s} / a \sigma_{b}$, is defined as the dimensionless ratio of surface (double-layer) conductivity $\sigma_{s}$ to bulk conductivity $\sigma_{b}$. Our analysis breaks down at $D u \approx 1$, which occurs when the total (equilibrium + induced) zeta potential reaches $\zeta \approx 2 k T / e \approx 50 \mathrm{mV}$ in most electrolytes. For uncharged conductors, this sets an upper bound on the voltage applied across the body by the applied field, $E_{b} a<50 \mathrm{mV}$. In such large electric fields, the double layer on the conducting body in regions of large zeta potential adsorbs enough neutral salt to perturb the bulk concentration (Bazant et al. 2004), which we neglect here in order to make the problem analytically tractable.

Assuming uniform bulk conductivity, the electrostatic potential $\Phi$ satisfies Laplace's equation (Ohm's law),

$$
\nabla^{2} \Phi=0
$$

everywhere outside the infinitesimally thin double layer around our ideally polarizable body. At the edge of the bulk region $\Gamma$, immediately adjacent to the body, a Neumann boundary condition expresses zero normal current,

$$
\hat{\boldsymbol{n}} \cdot \nabla \Phi(\boldsymbol{r})=0 \quad \text { for } \boldsymbol{r} \in \Gamma
$$

in the absence of tangential surface conduction or Faradaic electrochemical reactions. The far-field boundary condition,

$$
\Phi \sim \Phi_{a}=\Phi_{b}-\boldsymbol{E}_{b} \cdot \boldsymbol{r}-\frac{1}{2} \boldsymbol{G}_{b}: \boldsymbol{r} \boldsymbol{r}-\frac{1}{6} \boldsymbol{H}_{b}: \boldsymbol{r} \boldsymbol{r} \boldsymbol{r}-\ldots \text { as } r \rightarrow \infty,
$$

describes the applied potential $\Phi_{a}$ (and divergence-free electric field, $\boldsymbol{E}_{a}=-\nabla \Phi_{a}$ ) which would exist in the absence of the body. Here, $\Phi_{b}$ is the background potential; $E_{b}^{i}=-\partial \Phi_{a} / \partial r_{i}$ the background electric field (vector); $G_{b}^{i j}=-\partial^{2} \Phi_{a} / \partial r_{i} \partial r_{j}$ the background-field gradient matrix; $H_{b}^{i j k}=-\partial^{3} \Phi_{a} / \partial r_{i} \partial r_{j} \partial r_{k}$ the background-field second derivative tensor; etc.

In steady state, the zeta potential $\zeta$ of the double layer is simply the difference between the potential of the conductor $\Phi_{0}$ and the bulk potential just outside the double layer,

$$
\zeta(\boldsymbol{r})=\Phi_{0}-\Phi(\boldsymbol{r}) \text { for } \boldsymbol{r} \in \Gamma .
$$

For small zeta potentials $\zeta \ll k_{B} T / e, \zeta$ can be decomposed into two components,

$$
\zeta(\boldsymbol{r})=\zeta_{0}+\zeta_{i}(\boldsymbol{r})
$$

where $\zeta_{0}$ is constant, and $\zeta_{i}(\boldsymbol{r})$ is spatially varying, with $\int \zeta_{i} \mathrm{~d} A=0$. For a linear double-layer capacitance per unit area $C, \zeta_{0}$ is proportional to the total charge $Q_{0}$ on

$\dagger$ This dimensionless group was first discussed by J. J. Bikerman, but its fundamental importance in electrokinetics was first emphasized by S. S. Dukhin. Therefore, even though it is called ' $R e l$ ' in the Russian literature (Dukhin \& Shilov 1974; Dukhin 1993), we follow Lyklema (1995) in calling it the 'Dukhin number'. 
the body,

$$
Q_{0}=-C \int_{\Gamma} \zeta(\boldsymbol{r}) \mathrm{d} A=-A C \zeta_{0},
$$

where $A$ is the surface area of the conductor. In the case of a colloidal particle, the total charge $Q_{0}$ is fixed, and $\zeta_{0}$ represents the equilibrium zeta potential. In the context of microfluidics, the conductor's potential $\Phi_{0}$ relative to $\Phi_{b}$ (and thus $\zeta_{0}$ and $Q_{0}$ ) may also be controlled externally to drive 'fixed-potential ICEO' (Squires \& Bazant 2004). In both cases, the standard electro-osmotic/electrophoretic flows around a body with constant zeta potential $\zeta_{0}$ are well known. Instead, to focus on the spatial structure of ICEO flows, we will typically assume $Q_{0}=\zeta_{0}=0$.

\subsection{ICEO flow and ICEP motion}

Once the electrostatic problem has been solved, the ICEO flow is obtained by solving the Stokes equations,

$$
\eta \nabla^{2} \boldsymbol{u}-\nabla p=0, \quad \nabla \cdot \boldsymbol{u}=0,
$$

subject to zero normal fluid flux

$$
\hat{\boldsymbol{n}} \cdot \boldsymbol{u}(\boldsymbol{r})=0 \quad \text { for } \boldsymbol{r} \in \Gamma
$$

and to a (tangential) slip velocity given by (1.1),

$$
\boldsymbol{u}(\boldsymbol{r})=\boldsymbol{u}_{s}(\boldsymbol{r})=\frac{\varepsilon}{\eta} \zeta(\boldsymbol{r}) \nabla \Phi(\boldsymbol{r}) \quad \text { for } \boldsymbol{r} \in \Gamma
$$

at the surface of the conductor, just outside the double layer.

The boundary condition for the flow at infinity depends on the system studied. In the colloidal context, the induced-charge electrophoretic velocity $\boldsymbol{U}_{\text {ICEP }}$ and rotation $\boldsymbol{\Omega}_{\text {ICEP }}$ of the body are typically of interest, and are determined by requiring that there be no net force or torque on the body and imposing vanishing flows at infinity. This task is facilitated by an elegant set of relations which follows from the reciprocal theorem for Stokes flows (Stone \& Samuel 1996),

$$
\begin{aligned}
& \hat{\boldsymbol{F}} \cdot \boldsymbol{U}_{I C E P}=-\int \boldsymbol{u}_{s} \cdot \hat{\boldsymbol{\sigma}}_{F} \cdot \hat{\boldsymbol{n}} \mathrm{d} A, \\
& \hat{\boldsymbol{L}} \cdot \boldsymbol{\Omega}_{I C E P}=-\int \boldsymbol{u}_{s} \cdot \hat{\boldsymbol{\sigma}}_{L} \cdot \hat{\boldsymbol{n}} \mathrm{d} A,
\end{aligned}
$$

where $\boldsymbol{U}_{I C E P}$ is the translational velocity and $\boldsymbol{\Omega}_{\text {ICEP }}$ the angular velocity of a force-free and torque-free body on which a slip velocity $\boldsymbol{u}_{s}$ is specified. Here, $\hat{\boldsymbol{\sigma}}_{F}$ and $\hat{\boldsymbol{\sigma}}_{L}$ are the stress tensors due to complementary Stokes flow problems - respectively, the same object undergoing pure translation (with force $\hat{\boldsymbol{F}}$ ), and pure rotation (with torque $\hat{\boldsymbol{L}})$. General relations of the type (2.10)-(2.11) were first derived by Brenner (1964), and they have recently been applied to ICEP of asymmetric particles in uniform fields by Yariv (2005). In the special case of spherical bodies, Stone \& Samuel (1996) have noted that these relations reduce to simple formulae for the linear and angular velocity,

$$
\begin{aligned}
& \boldsymbol{U}_{\text {ICEP }}=-\frac{1}{4 \pi} \int \boldsymbol{u}_{s}(\theta) \mathrm{d} \Omega, \\
& \boldsymbol{\Omega}_{\text {ICEP }}=-\frac{3}{8 \pi a} \int \hat{\boldsymbol{r}} \times \boldsymbol{u}_{s}(\theta) \mathrm{d} \Omega,
\end{aligned}
$$

where $\mathrm{d} \Omega$ is an element of solid angle. 
Microfluidic ICEO systems, on the other hand, typically involve the ICEO flow around structures that are held in place. In that case, the above strategy is modified by simply superposing two flows: (i) (force-free) ICEP as described above, and (ii) the flow around the body held fixed in an equal and opposite flow $\boldsymbol{u}_{\infty}=\boldsymbol{U}_{\text {ICEP }}+\boldsymbol{\Omega}_{\text {ICEP }} \times \boldsymbol{r}$. Two-dimensional Stokes flows around forced cylinders diverge at infinity (the socalled 'Stokes paradox', addressed by Proudman \& Pearson 1957), complicating this approach. In practical situations, this divergence is cut off at some length scale, such as the cylinder length (at which point the flow becomes three-dimensional), the distance to a nearby solid surface, or the inertial length scale $a / R e$.

The approach we adopt here is to calculate the ICEP flow (which, to leading order, is independent of the system geometry), with the understanding that ICEO around fixed cylindrical bodies will require the mobility problem to be solved for the particular system of interest. The Stokes flow around an infinite cylinder translating towards or along a solid planar wall located a distance $d$ away, for example, is well-posed, and has been treated by Jeffrey \& Onishi (1981). Nevertheless, the ICEP rotation and velocity must still be determined for cylindrical bodies. Fortunately, and perhaps remarkably, (2.10) and (2.11) hold for two-dimensional bodies, despite the logarithmic divergence that occurs for forced two-dimensional Stokes flows. For circular cylinders, the simplified formulae read

$$
\begin{array}{r}
\boldsymbol{U}_{I C E P}=-\frac{1}{2 \pi} \int_{0}^{2 \pi} \boldsymbol{u}_{s}(\theta) \mathrm{d} \theta, \\
\boldsymbol{\Omega}_{I C E P}=-\frac{1}{2 \pi a} \int_{0}^{2 \pi} \hat{\boldsymbol{r}} \times \boldsymbol{u}_{s}(\theta) \mathrm{d} \theta .
\end{array}
$$

We discuss the subtleties in the Appendix.

\subsection{Dielectrophoresis and electrorotation}

A non-uniform background electric field generally exerts an electrostatic force and torque on a polarizable solid body, whether or not ICEO fluid slip occurs at the surface. In a microfluidic device, this force and torque, in addition to viscous interactions with the walls, must be opposed in order to hold the body fixed in place while driving ICEO flow. For a colloidal particle, the electrostatic force and torque cause dielectrophoresis (DEP) and electrorotation, respectively (Pohl 1978), in addition to the force-free and torque-free ICEP motion. As we shall see, the competition between DEP and ICEP is rather subtle, since the two effects act in opposite directions with similar magnitude for ideally polarizable bodies.

The electrostatic force derives from the action of the non-uniform applied field on the induced charge distribution, typically characterized by low-order multipole moments for an isolated body. These moments appear as coefficients of the far-field expansion of the electrostatic potential (Jackson 1975):

$$
(4 \pi \varepsilon)\left(\Phi-\Phi_{a}\right) \sim \frac{\tilde{Q}_{0}}{r}+\frac{\boldsymbol{p} \cdot \boldsymbol{r}}{r^{3}}+\frac{1}{2} \frac{\boldsymbol{Q}: \boldsymbol{r} \boldsymbol{r}}{r^{5}}+\ldots \quad \text { as } r \rightarrow \infty
$$

in three dimensions, or

$$
(2 \pi \varepsilon)\left(\Phi-\Phi_{a}\right) \sim \tilde{Q}_{0} \ln r+\frac{\boldsymbol{p} \cdot \boldsymbol{r}}{r^{2}}+\frac{1}{2} \frac{\boldsymbol{Q}: \boldsymbol{r} \boldsymbol{r}}{r^{4}}+\ldots \text { as } r \rightarrow \infty
$$

in two dimensions, where $\tilde{Q}_{0}$ is the monopole moment (net charge), $\boldsymbol{p}$ is the dipole moment induced by the applied field $\boldsymbol{E}_{b} ; \boldsymbol{Q}$ is the quadrupole moment induced by the applied field gradient $\boldsymbol{G}_{b}$; etc. Note that the multipole moments also reflect ionic 
screening of the 'bare' moments of the charge distribution on the body, which would exist in the absence of the electroylte. Here, we consider conductors and dielectrics without any fixed charges, so we have only the bare total charge $Q_{0}$, which is typically screened to give $\tilde{Q}_{0}=0$, although $\tilde{Q}_{0} \neq 0$ is possible out of equilibrium in fixedpotential ICEO (Squires \& Bazant 2004). Since the leading induced term in the far field is a dipole, much attention has focused on calculating the induced dipole moment of dielectric and conducting colloids, especially in the Russian literature (Dukhin \& Shilov 1980). Higher-order induced multipoles have recently been considered in the classical context of DEP in non-conducting liquids (Washizu \& Jones 1994; Jones \& Washizu 1996; Wang, Wang \& Gascoyne 1997), but we are not aware of any prior work on general applied fields in electrolytes, also accounting for ICEO flow.

The total force and torque on any volume of the fluid are conveniently given in terms of the stress tensor, $\boldsymbol{\sigma}$, by

$$
\begin{gathered}
\boldsymbol{F}=\int \boldsymbol{\sigma} \cdot \hat{\boldsymbol{n}} \mathrm{d} A, \\
\boldsymbol{L}=\int \boldsymbol{r} \times \boldsymbol{\sigma} \cdot \hat{\boldsymbol{n}} \mathrm{d} A .
\end{gathered}
$$

The stress tensor contains contributions from osmotic, electrical and viscous stresses on the fluid, $\boldsymbol{\sigma}=-p \boldsymbol{I}+\boldsymbol{\sigma}_{M}+\boldsymbol{\sigma}_{v}$, where

$$
\begin{aligned}
\boldsymbol{\sigma}_{M} & =-(\varepsilon / 2) E^{2} \boldsymbol{I}+\varepsilon \boldsymbol{E} \boldsymbol{E}, \\
\boldsymbol{\sigma}_{v} & =\eta\left(\nabla \boldsymbol{u}+(\nabla \boldsymbol{u})^{T}\right),
\end{aligned}
$$

are the Maxwell and viscous stress tensors, respectively (Russel et al. 1989; Squires \& Bazant 2004; Yariv 2005).

To remove any confusion due to ICEP, we work in a reference frame that translates and rotates with the ICEP velocity and rotation of the particle. Since ICEP is free of force and torque, it will not contribute to (2.18)-(2.19). To prevent the body from translating or rotating within this frame, we apply a force and torque on the body to counteract the DEP force and torque. Thus (2.18)-(2.19) give the DEP force and torque, where any surface of integration that encloses the body may be chosen owing to mechanical equilibrium, $\boldsymbol{\nabla} \cdot \boldsymbol{\sigma}=\mathbf{0}$. We choose the surface at infinity, where the ionic concentrations are constant and viscous stresses decay quickly enough to be negligible, leaving only the far-field electrical stresses. In this limit, the stress tensor reduces to the standard Maxwell tensor for electrostatics $\boldsymbol{\sigma}_{M}$.

The integrals (2.18)-(2.19) may thus be evaluated using the far-field expansions of the applied potential (2.3) and the induced multipoles (2.16)-(2.17) to obtain

$$
\begin{aligned}
\boldsymbol{F} & =\tilde{Q}_{0} \boldsymbol{E}_{b}+\boldsymbol{p} \cdot \boldsymbol{G}_{b}+\alpha \boldsymbol{Q}: \boldsymbol{H}_{b}+\ldots, \\
\boldsymbol{L} & =\boldsymbol{p} \times \boldsymbol{E}_{b}+\ldots,
\end{aligned}
$$

where $\alpha=1 / 6$ in three dimensions and $\alpha=1 / 4$ in two dimensions, following Wang et al. (1997). The classical DEP force, $\boldsymbol{F}_{D E P}=\boldsymbol{p} \cdot \boldsymbol{G}_{b}$, and torque, $\boldsymbol{L}_{D E P}=\boldsymbol{p} \times \boldsymbol{E}_{b}$, are associated with only the induced dipole moment $\boldsymbol{p}$. Note again that $\tilde{Q}_{0}$ in $(2.22)$ reflects the 'net' charge as seen in the far field, which almost always vanishes owing to double-layer screening of the bare charge $Q_{0}$. The same expansion can also be obtained from a dyadic tensor representation of the multipolar moments (Washizu \& Jones 1994; Jones \& Washizu 1996).

Having mentioned standard electro-osmosis, ICEO, and dielectrophoresis and its relatives, we briefly mention other effects that arise in such systems, discussed more 
extensively by Morgan \& Green (2003) and Ramos et al. (1998). Electrothermal flows occur when viscous (Joule) heating causes thermal (and thus permittivity) gradients that couple with the electric fields to give rise to further Maxwell stresses. Thermal gradients can also give rise to buoyancy-driven flows. In our previous work (Squires \& Bazant 2004), we briefly discussed charge convection (significant Péclet numbers), as well as such high- $\zeta$ effects as surface conduction (significant Dukhin numbers) and electrochemical reactions (Faradaic currents). For simplicity, we will neglect such effects here.

We have now built up the machinery necessary to treat the steady-state behaviour of arbitrarily shaped conducting particles immersed in an electrolytic fluid and subject to an applied electric field. Below, we treat four paradigmatic examples for ICEO in systems that break spatial symmetry in some way. In all cases, we pursue the following general strategy: (i) we find the steady-state electric field, which obeys Laplace's equation (2.1) subject to the no-flux boundary condition (2.2); (ii) we find the induced zeta potential using (2.4), and enforce the total charge condition (2.6); (iii) we find the slip velocity $\boldsymbol{u}_{s}$ from (2.9); (iv) we solve the Stokes equations (or, equivalently, use $\boldsymbol{u}_{s}$ in (2.14) or (2.15) to obtain the ICEP linear and rotational velocity); and (v) determine the DEP force and torque using (2.22) and (2.23), and the DEP motion that results.

\section{Conductors with inhomogeneous surface properties}

\subsection{Partial dielectric or insulating coatings}

We now begin our treatment of specific examples of ICEO systems that break spatial symmetry in some way. Our first example is perhaps the simplest mathematically and the clearest intuitively: a symmetric (spherical or cylindrical) conductor whose surface properties are inhomogeneous. For example, a conductor could be partially coated with a dielectric layer that is thin enough not to change the shape appreciably, but thick enough to suppress ICEO flow locally. That the flow is suppressed is demonstrated in our earlier work (Squires \& Bazant 2004): when the potential drop between the conducting surface and the bulk electric field occurs over both the induced double layer and the dielectric layer (thickness $\lambda_{d}$, permittivity $\varepsilon_{d}$ ), the extra capacitance of the dielectric layer reduces the induced zeta potential to

$$
\zeta_{i}=\frac{\Phi_{0}-\Phi_{\Gamma}}{1+\varepsilon \lambda_{d} / \varepsilon_{d} \lambda_{D}} \approx \frac{\lambda_{D}}{\lambda_{d}} \frac{\varepsilon_{d}}{\varepsilon}\left(\Phi-\Phi_{0}\right),
$$

with the rest of the potential drop $\Phi-\Phi_{0}$ occurring across the coating itself. For sufficiently thick dielectric layers, the ICEO slip velocity (which varies with $\zeta_{i}$ ) is reduced by a factor of $O\left(\lambda_{D} / \lambda_{d}\right)$. For simplicity, we will assume the dielectric coating to be thick enough to render any induced charge (and therefore ICEO slip velocity) negligible.

A suitable example of such partially conducting bodies are the 'magnetically modulated optical nanoprobes (MagMOONs)' described by Anker \& Kopelman (2003), which are magnetic colloidal spheres upon which a thin metal film is evaporatively deposited on one hemisphere. The magnetic moment of MagMOONs is not necessary for the present discussion, although it would clearly allow another avenue for manipulation. Another example involves 'nanobarcodes' (Nicewarner-Pena et al. 2001; Finkel et al. 2004), which are cylindrical rods composed of alternating metallic nanolayers (silver/gold), used to store information in a colloid or to 'tag' biomolecules. Prior to optically 'reading' nanobarcodes in a colloid, they are aligned by an electric field, 

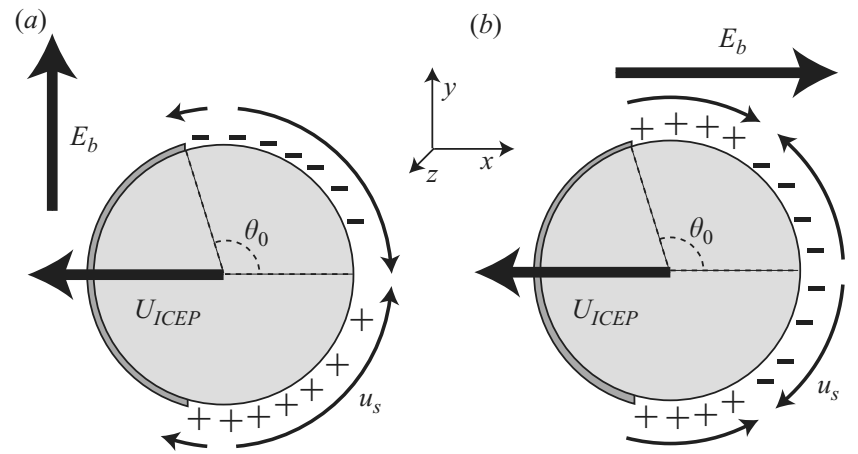

FIGURE 2. Induced-charge electrophoretic motion of partially coated cylinders. $(a)$ A cylinder with a partial dielectric coating that breaks left-right symmetry, and $(b)$ a cylinder whose partial dielectric coating breaks fore-aft symmetry. Such partially coated cylinders, if freely suspended, experience an ICEP motion in the direction of their coated ends, whether in AC or DC applied fields. Partially coated conducting cylinders that are held fixed in place act to pump fluid in the direction away from the coated portion of the cylinder.

and they can also be manipulated by DEP; our analysis shows that ICEO and ICEP can play important roles in these processes. Theoretical and experimental studies of the ICEP of metallic rods are underway (Rose \& Santiago 2006; Saintillan, Darve \& Shaqfeh 2006).

The clearest and most straightforward example involves a half-coated cylinder with its symmetry axis oriented perpendicular to the field (that is, left-right asymmetric, as in figure $2 a$ ). The fore-aft orientation (figure $2 b$ ) then follows, and introduces an additional complexity - charge-conservation must be enforced (equation (2.6)). We then present the general case where an arbitrary amount of the cylinder is coated, and it is oriented in an arbitrary direction with respect to the field. We conclude by presenting the analogous results for partially coated spheres.

In general, we consider a cylinder whose surface is metallic for angles $|\theta|<\theta_{0}$ (that is, it is coated in the range $\theta_{0}<\theta<2 \pi-\theta_{0}$ ). An electric field is applied 'at infinity' at some angle $\gamma$; when $\gamma=0$, the cylinder is fore-aft asymmetric, and when $\gamma= \pm \pi / 2$, it is left-right asymmetric. The $\hat{\boldsymbol{x}}$-axis points along $\theta=0$, and $\hat{\boldsymbol{y}}$ and $\hat{\boldsymbol{z}}$ complete a standard right-handed Cartesian coordinate system, with the electric field applied in the $(\hat{\boldsymbol{x}}, \hat{\boldsymbol{y}})$, plane.

For cylinders, the bulk electrostatic potential $\Phi$ is given by

$$
\Phi=-E_{b} \cos (\theta-\gamma)\left(r+\frac{a^{2}}{r}\right),
$$

giving a tangential field

$$
\boldsymbol{E}_{\|}=-2 E_{b} \sin (\theta-\gamma) \hat{\boldsymbol{\theta}} .
$$

\subsection{Cylinder with left-right asymmetric coating}

We consider first a left-right asymmetric half-coated cylinder $\left(\theta_{0}=\pi / 2\right)$, where the field angle is $\gamma=\pi / 2$ (figure $2 a$ ). Using (2.4) and (3.2), the induced zeta potential is given by

$$
\zeta_{i}(|\theta|<\pi / 2)=2 E_{b} a \sin \theta,
$$

which naturally obeys the no-charge condition, (2.6). Note that this is the same zeta potential for standard (symmetric) ICEO over the metallic portion, but $\zeta_{i}=0$ over 
(a)

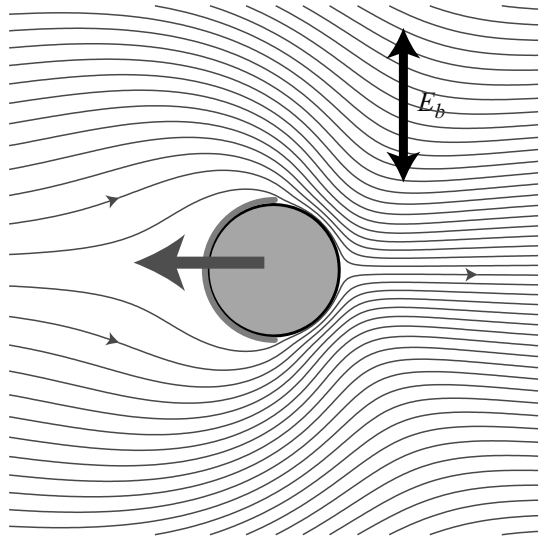

(b)

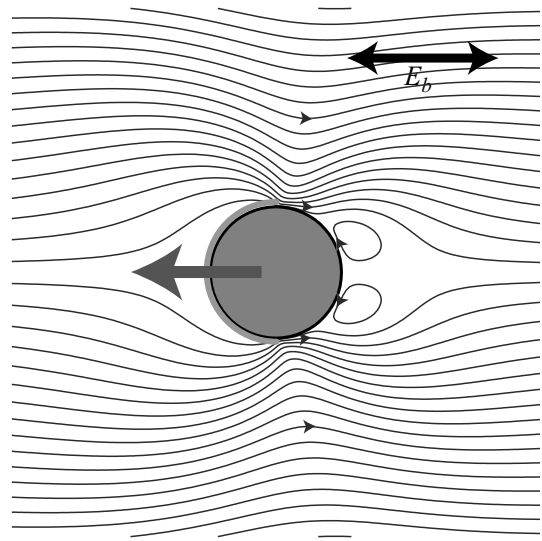

FIGURE 3. Streamlines (in the co-moving frame) for the ICEO flow around a conducting cylinder whose left-hand side is coated with a dielectric layer that suppresses ICEO flow. Regardless of whether the cylinder asymmetry is $(a)$ left-right or $(b)$ fore-aft with respect to the field, a freely suspended partially coated cylinder moves in the direction of its coated end.

the coated portion. The slip velocity is therefore given by

$$
\boldsymbol{u}_{s}(|\theta|<\pi / 2)=-2 U_{0} \sin 2 \theta \hat{\boldsymbol{\theta}},
$$

as shown in figure 2(a). Equation (2.14) gives an ICEP velocity

$$
\boldsymbol{U}=-\frac{4}{3 \pi} U_{0} \hat{\boldsymbol{x}} \approx-0.42 U_{0} \hat{\boldsymbol{x}}
$$

in the direction of the coated end. According to (2.15), the cylinder does not rotate (as expected from symmetry). Streamlines for the ICEO flow around a halfcoated conducting cylinder oriented in a left-right asymmetric fashion are shown in figure $3(a)$.

\subsection{Cylinder with fore-aft asymmetric coating}

Secondly, we consider a fore-aft asymmetric cylinder $(\gamma=0)$, as shown in figure $2(b)$. Using (2.4), (2.6) and (3.2), the induced zeta potential is found to be

$$
\zeta_{i}(|\theta|<\pi / 2)=2 E_{b} a\left(\cos \theta-\frac{2}{\pi}\right),
$$

and zero elsewhere. Note the second term is required to satisfy the no-charge condition (equation (2.6)). The slip velocity is therefore given by

$$
\boldsymbol{u}_{s}(|\theta|<\pi / 2)=4 U_{0} \sin \theta\left(\cos \theta-\frac{2}{\pi}\right) \hat{\boldsymbol{\theta}},
$$

which, using (2.14), gives an ICEP velocity

$$
\boldsymbol{U}=-\frac{2}{3 \pi} U_{0} \hat{\boldsymbol{x}} \approx 0.21 U_{0} \hat{\boldsymbol{x}} .
$$

Streamlines for the ICEO flow around a half-coated conducting cylinder oriented fore-aft with respect to the field are shown in figure $3(b)$.

\subsection{General direction and coating}

Finally, we present results for general field angle $\gamma$ and coating $\theta_{0}$. The approach is analogous, and gives an ICEP velocity for a freely suspended, asymmetrically coated 
sphere,

$U_{x}=-\frac{U_{0}}{\pi}\left[\frac{3 \sin \theta_{0}+\sin 3 \theta_{0}}{3}+\frac{\cos 3 \theta_{0}-\cos \theta_{0}}{2 \theta_{0}}+\sin ^{2} \gamma\left(\frac{\cos \theta_{0}-\cos 3 \theta_{0}}{2 \theta_{0}}-\frac{2 \sin 3 \theta_{0}}{3}\right)\right]$,

which is always negative (directed towards the coated end). In addition, however, the ICEP velocity has a non-zero velocity perpendicular to the asymmetry axis, given by

$$
U_{y}=\frac{U_{0}}{6 \pi} \sin 2 \gamma\left(2 \sin 3 \theta_{0}+\frac{3 \cos 3 \theta_{0}-3 \cos \theta_{0}}{2 \theta_{0}}\right) .
$$

The term in parentheses is negative for $\theta_{0}<0.61 \pi$, after which it switches sign meaning that the transverse ICEP velocity occurs in either direction, depending on the field angle $\gamma$ and the coating angle $\theta_{0}$. Lastly, using (2.15), we find the rotation speed of the asymmetrically coated cylinder to be

$$
\boldsymbol{\Omega}=\frac{U_{0}}{\pi a} \sin 2 \gamma\left(\sin 2 \theta_{0}-\frac{1-\cos 2 \theta_{0}}{\theta_{0}}\right) \hat{z},
$$

from which it is evident that the fore-aft asymmetric orientation is unstable to rotations, and the left-right asymmetric orientation is stable. Because the ICEO velocity scale $U_{0}$ varies linearly with $a$, the rotation rate is independent of cylinder radius $a$.

\subsection{Partially coated conducting spheres}

Finally, we consider the analogous situation for a sphere coated for polar angles $|\theta|>\theta_{0}$, and subjected to an electric field with magnitude $\alpha E_{b}$ in the $\theta=0$ (or $\hat{\boldsymbol{x}}$ ) direction, along with a transverse field of strength $\beta E_{b}$ in the $\theta=\pi / 2, \phi=0$ (or $\hat{\boldsymbol{y}}$ ) direction.

In spherical coordinates, the potential is

$$
\Phi=-E_{b}\left(r+\frac{a^{3}}{2 r^{2}}\right)(\alpha \cos \theta+\beta \sin \theta \cos \phi),
$$

giving an induced zeta potential

$$
\zeta_{i}=\zeta_{c}+\frac{3}{2} E_{b} a(\alpha \cos \theta+\beta \cos \phi \sin \theta),
$$

where $\zeta_{c}$ satisfies the total charge constraint, and is given by

$$
\zeta_{c}=-\frac{3}{4} \alpha E_{b} a\left(1+\cos \theta_{0}\right) \text {. }
$$

The sphere moves with velocity

$$
\begin{aligned}
& U_{x}=-\frac{3}{64} U_{0}\left(32 \alpha^{2} \cos ^{2} \frac{\theta_{0}}{2} \sin ^{6} \frac{\theta_{0}}{2}+3 \beta^{2} \sin ^{4} \theta_{0}\right), \\
& U_{y}=-\frac{3}{4} U_{0} \alpha \beta \cos ^{2} \frac{\theta_{0}}{2} \sin ^{4} \frac{\theta_{0}}{2}\left(1+2 \cos \theta_{0}\right), \\
& U_{z}=0,
\end{aligned}
$$

and rotates with velocity

$$
\Omega_{z}=-\frac{27}{8} \frac{U_{0}}{a} \alpha \beta \cos ^{2} \frac{\theta_{0}}{2} \sin ^{4} \frac{\theta_{0}}{2},
$$




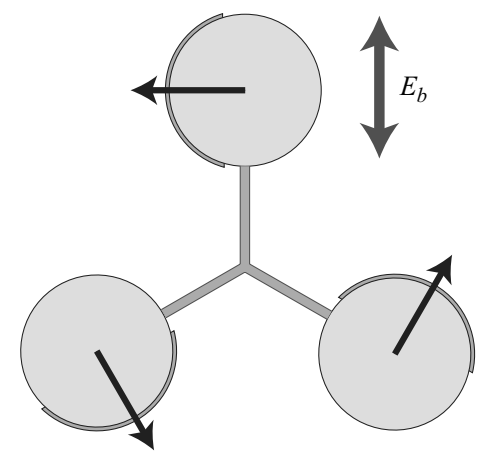

FIGURE 4. Combining multiple partially coated spheres into a composite object, where the coated ends are directed in the same sense around the circle, yields a structure that rotates under any applied field. Here the 'connector' is electrically insulating.

about the $\hat{z}$-axis. Note that like the coated cylinder, the coated sphere always moves towards the coated end $\left(U_{x}<0\right.$ for all $\alpha, \beta$ and $\left.\theta_{0}\right)$. Also, like the coated cylinder, the fore-aft orientation is unstable and the left-right orientation is stable.

\subsection{Ever-rotating structures}

Finally, we discuss an interesting consequence of the above results: since partially coated symmetric conductors generically 'swim' towards the coated end, we can design objects that rotate steadily under AC or DC electric fields. Figure 4 shows a structure composed of multiple partially coated conducting bodies connected with insulating 'spokes' of length $d$, oriented so that the coated end 'points' in the same sense around a circle. An AC electric field, applied in any direction, would give rise to an ICEP motion of the conductors, each of which would contribute to a net rotation of the body as a whole.

The rotation rates can be calculated as follows: we assume the partially coated spheres to be located far enough apart that they do not interact hydrodynamically or electrostatically. Each sphere would have some ICEP velocity if freely suspended, whereas the 'spokes' exert forces on each (parallel and perpendicular to each rod) to ensure the ensemble moves as a rigid body. A composite spinner composed of two half-coated $\left(\theta_{0}=\pi / 2\right)$ spheres would rotate with a velocity,

$$
\Omega_{2}=\frac{3}{128} \frac{U_{0}}{d}(5-\cos 2 \gamma),
$$

that varies with the angle $\gamma$ of the spinner relative to the electric field. (Note, however, that a two-sphere composite with $\theta_{0}=\pi-\sin ^{-1}(\sqrt{3 / 8})$ coating would rotate with a steady velocity.) A composite spinner composed of three or more half-coated spheres would rotate with a steady velocity,

$$
\Omega_{3^{+}}=\frac{15}{128} \frac{U_{0}}{d}
$$

Furthermore, since the left-right asymmetric orientation is stable, such composite bodies will naturally rotate to orient themselves perpendicular to the applied field.

One could imagine various uses for ICEO spinners - because such structures rotate whenever an electric field (AC or DC) is present, they could obviously be used as electric field sensors. They could also be used in single-molecule experiments to apply a given torque to a biomolecule. Or, from a biomimetic standpoint, ICEO spinners are analogous to rotary motor proteins, such as those that drive bacterial flagellar 
rotation (Berg 2003) and $F_{1}$ ATP-ase (Kinosita, Adachi \& Itoh 2004), and represent simple rotary motors.

\section{Nearly symmetric conducting bodies}

\subsection{ICEO flows around near-cylinders}

The next example we consider involves conducting bodies whose shapes, rather than surface properties, are asymmetric. Specifically, we consider conductors that are nearly symmetric, but whose shape is perturbed slightly in an arbitrary asymmetric fashion. While, strictly speaking, the shape asymmetry must be slight for these results to hold, we expect the qualitative results to hold for more highly asymmetric shapes. Such highly asymmetric systems would need to be treated numerically, whereupon the results of Yariv (2005) could be used. Here, we treat 'nearly cylindrical' bodies and follow with analogous 'near-spheres'.

Specifically, we consider a cylindrical body with perturbed radius

$$
R=a[1+\epsilon f(\theta)],
$$

where $\epsilon$ is a small parameter and $\theta=0$ along the $\hat{\boldsymbol{x}}$-axis. The vectors normal and tangent to the surface are given by

$$
\begin{gathered}
\hat{\boldsymbol{n}}=\hat{\boldsymbol{r}}-\epsilon f_{\theta} \hat{\boldsymbol{\theta}}+O\left(\epsilon^{2}\right), \\
\hat{\boldsymbol{t}}=\hat{\boldsymbol{\theta}}+\epsilon f_{\theta} \hat{\boldsymbol{r}}+O\left(\epsilon^{2}\right),
\end{gathered}
$$

where $f_{\theta}=\partial f / \partial \theta$. While the method presented here applies to arbitrary perturbations, we will specifically consider the simplest symmetry-breaking perturbation

$$
f(\theta)=P_{3}(\cos \theta),
$$

representing a near-cylinder that 'points' in the positive $\hat{x}$-direction. A constant electric field, directed along the angle $\gamma$, is applied 'at infinity': when $\gamma=0$, the body is foreaft asymmetric (figure $5 a$ ) with respect to the field, and when $\gamma=\pi / 2$, the body is left-right asymmetric (figure $5 b$ ).

As above, we determine first the steady-state electric field, from which the induced zeta potential and slip velocity follow. We then solve the steady Stokes equations with specified slip velocity. The advantage to treating 'nearly' symmetric bodies is that the boundary itself can be treated perturbatively (see, e.g. Hinch 1991, pp. 46 47), giving a set of effective boundary conditions that are applied on the simpler (symmetric) boundary, rather than on the original (complicated) boundary. In so doing, the problem can be solved and the first effects of shape asymmetry can be studied.

\subsubsection{Electric field}

We decompose the electric potential $\Phi$ into background and induced components, $\Phi=\Phi_{b}+\Phi_{i}$, where

$$
\Phi_{b}=-E_{b} r \cos (\theta-\gamma) .
$$

The induced component $\Phi_{i}$ obeys Laplace's equation (2.1) with boundary conditions

$$
\Phi_{i}(r \rightarrow \infty) \rightarrow 0 \text { and }\left.\hat{\boldsymbol{n}} \cdot \nabla \Phi_{i}\right|_{r=R}=-\left.\hat{\boldsymbol{n}} \cdot \nabla \Phi_{b}\right|_{r=R},
$$

from (2.3) and (2.2).

To find an approximate solution for the electric field, we use a boundary perturbation posing an expansion $\Phi_{i}=\Phi_{0}+\epsilon \Phi_{1}+O\left(\epsilon^{2}\right)$. Using (4.2) to expand the boundary 
(a)

(c)

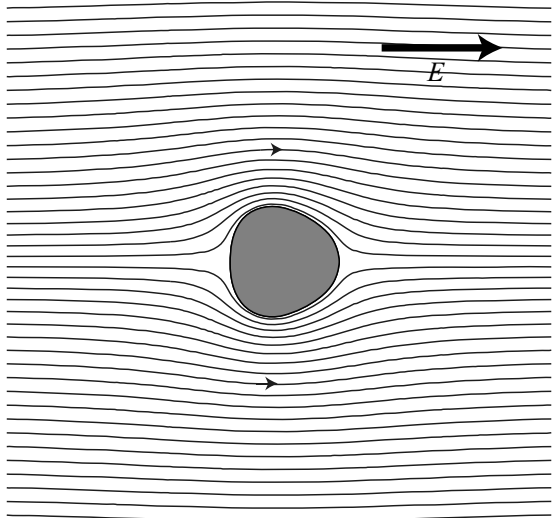

(b)

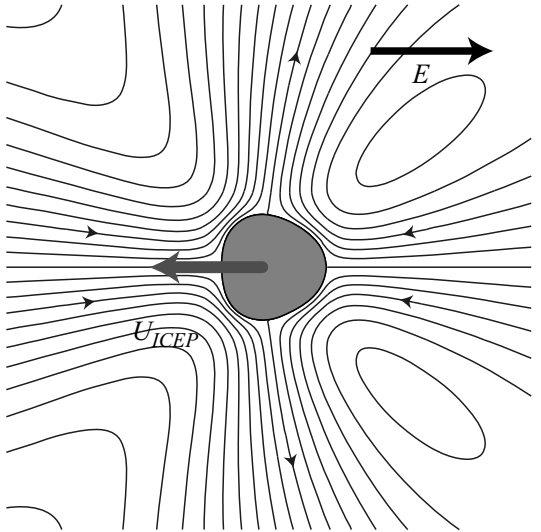

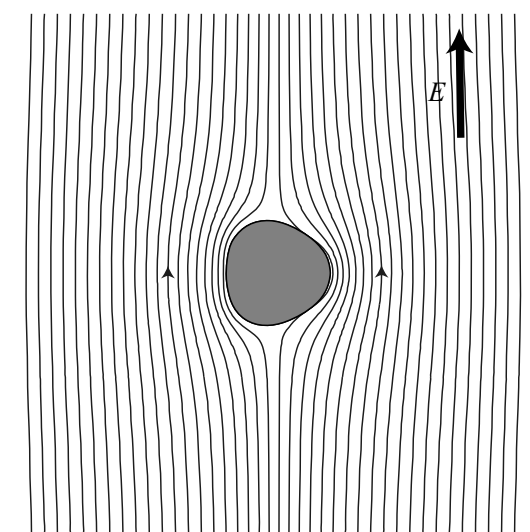

$(d)$

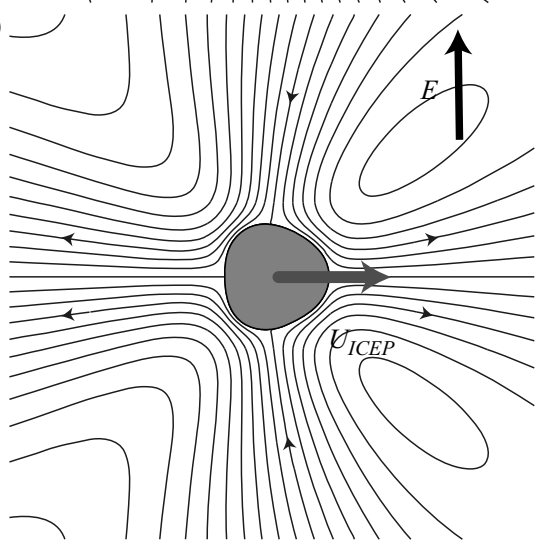

FIGURE 5. Two-dimensional asymmetric conductors in uniform (DC or AC) applied electric fields. $(a)$ The electric field lines and $(b)$ streamlines (in the co-moving frame) of the ICEO flow around a near-cylinder with broken fore-aft symmetry, with $R(\theta)=a\left[1+\epsilon P_{3}(\cos \theta)\right]$, which, if free, would move by ICEP towards its blunt end. $(c)$ The electric field and $(d)$ the ICEO flow for broken left-right symmetry, with $R=a\left[1+\epsilon P_{3}(\sin \theta)\right]$, which would move by ICEP towards its sharp end. Here $\epsilon=0.1$.

conditions, we require the fields to obey

$$
\begin{aligned}
& \left.\hat{\boldsymbol{r}} \cdot \nabla \Phi_{0}\right|_{a}=-\left.\hat{\boldsymbol{r}} \cdot \nabla \Phi_{b}\right|_{r=a}, \\
& \left.\hat{\boldsymbol{r}} \cdot \nabla \Phi_{1}\right|_{a}=\left[f_{\theta} \hat{\boldsymbol{\theta}} \cdot \nabla\left(\Phi_{0}+\Phi_{b}\right)-a f \partial_{r r}\left(\Phi_{0}+\Phi_{b}\right)\right]_{r=a} .
\end{aligned}
$$

The leading-order field is given by

$$
\Phi_{0}=-E_{b} \frac{a^{2}}{r} \cos (\theta-\gamma)
$$

from which it follows that the first-order correction obeys

$$
\left.\frac{\partial \Phi_{1}}{\partial r}\right|_{a}=2 E_{b} \frac{\partial}{\partial \theta}[f(\theta) \sin (\theta-\gamma)] .
$$

Straightforward manipulations give the $O(\epsilon)$ correction for $f=P_{3}(\cos \theta)$ to be

$$
\Phi_{1}=\frac{a^{3}}{8 r^{2}} E_{b}[5 \cos (2 \theta+\gamma)-3 \cos (2 \theta-\gamma)]-\frac{5 a^{5}}{8 r^{4}} E_{b} \cos (4 \theta-\gamma) .
$$


Note that the dipolar component of the induced electric field (equation (4.9)) is aligned with the applied field, and thus no DEP torque is exerted. Furthermore, no DEP force is exerted owing to the absence of a gradient in the applied electric field.

The induced zeta potential $\zeta_{i}$ is then given by (2.4) to be

$$
\frac{\zeta_{i}(\theta)}{E_{b} a}=2 \cos (\theta-\gamma)+\frac{\epsilon}{8}[-3 \cos \gamma+5 \cos (4 \theta-\gamma)+3 \cos (2 \theta-\gamma)-5 \cos (2 \theta+\gamma)] \text {. }
$$

Here the constant term $(-3 \epsilon / 8 \cos \gamma)$ has been introduced to satisfy the no-charge boundary condition (2.6), which is given to $O(\epsilon)$ by

$$
\int_{0}^{2 \pi} \zeta_{i}(1+\epsilon f(\theta)) \mathrm{d} \theta=0
$$

where we have used the arclength $\mathrm{d} l=\sqrt{R^{2} \mathrm{~d} \theta^{2}+\mathrm{d} r^{2}}=R \mathrm{~d} \theta+O\left(\epsilon^{2}\right)$.

\subsubsection{Fluid flow}

The fluid velocity obeys the steady Stokes equations (2.7) with solutions that decay far from the body, admit no normal flow (2.8) at the surface $R$, and with tangential boundary condition (2.9) satisfied on the surface $R$ by the Smoluchowski slip velocity $\boldsymbol{u}^{s}$

where

$$
\boldsymbol{u}^{s}(\theta)=\left.\frac{\varepsilon}{\eta} \zeta_{i} \nabla \Phi\right|_{r=R}=\left[u_{0}^{s}(\theta)+u_{1}^{s}(\theta)\right] \hat{\boldsymbol{t}}
$$

$$
\begin{gathered}
u_{0}^{s}=2 U_{0} \sin 2(\theta-\gamma), \\
u_{1}^{s}=\frac{U_{0}}{4}[-3 \sin (\theta-2 \gamma)+3 \sin (3 \theta-2 \gamma)+10 \sin (5 \theta-2 \gamma)] .
\end{gathered}
$$

We pose an expansion for the fluid velocity, $\boldsymbol{u}=\boldsymbol{u}_{0}+\epsilon \boldsymbol{u}_{1}+\ldots$ and obtain the leading-order fluid-flow boundary conditions

$$
\left.\hat{\boldsymbol{\theta}} \cdot \boldsymbol{u}_{0}\right|_{a}=u_{0}^{s}(\theta),\left.\quad \hat{\boldsymbol{r}} \cdot \boldsymbol{u}_{0}\right|_{a}=0
$$

which are solved by

$$
\begin{aligned}
& \hat{\boldsymbol{r}} \cdot \boldsymbol{u}_{0}=\frac{2 a\left(a^{2}-r^{2}\right)}{r^{3}} U_{0} \cos 2(\theta-\gamma), \\
& \hat{\boldsymbol{\theta}} \cdot \boldsymbol{u}_{0}=\frac{2 a^{3}}{r^{3}} U_{0} \sin 2(\theta-\gamma),
\end{aligned}
$$

as described by Squires \& Bazant (2004).

Finally, the boundary conditions for $\boldsymbol{u}_{1}$ are given by

$$
\begin{aligned}
& \left.\hat{\boldsymbol{\theta}} \cdot \boldsymbol{u}_{1}\right|_{a}=u_{1}^{s}(\theta)-a f \frac{\partial}{\partial r}\left[\hat{\boldsymbol{\theta}} \cdot \boldsymbol{u}_{0}\right]_{r=a}, \\
& \left.\hat{\boldsymbol{r}} \cdot \boldsymbol{u}_{1}\right|_{a}=\left[f_{\theta} \hat{\boldsymbol{\theta}} \cdot \boldsymbol{u}_{0}-a f \frac{\partial}{\partial r}\left(\hat{\boldsymbol{r}} \cdot \boldsymbol{u}_{0}\right)\right]_{r=a},
\end{aligned}
$$

where we have used (4.2) and (4.3). For $f(\theta)=P_{3}(\cos \theta)$, the boundary conditions are given by

$$
\left.\hat{\boldsymbol{\theta}} \cdot \boldsymbol{u}_{1}\right|_{a}=\frac{U_{0}}{8}[-15 \sin (\theta+2 \gamma)+3 \sin (\theta-2 \gamma)+15 \sin (3 \theta-2 \gamma)+35 \sin (5 \theta-2 \gamma)],
$$




$$
\left.\hat{\boldsymbol{r}} \cdot \boldsymbol{u}_{1}\right|_{a}=\frac{U_{0}}{8}[-5 \cos (\theta+2 \gamma)+3 \cos (\theta-2 \gamma)+9 \cos (3 \theta-2 \gamma)+25 \cos (5 \theta-2 \gamma)] \text {. }
$$

To summarize, the above approach takes a flow defined on a non-trivial boundary, and expresses equivalent boundary conditions over a simple cylinder of radius $a$. We can now determine the ICEP velocity of the near-cylinder without solving for the flow field, by simply using (2.14). The $O(1)$ slip velocity is symmetric and results in no ICEP. We express the $O(\epsilon)$ slip velocity equations (4.22)-(4.23) in Cartesian components,

$$
\left.\boldsymbol{u}_{1}\right|_{a}=\left.\left(\left(-\hat{\boldsymbol{\theta}} \cdot \boldsymbol{u}_{1} \sin \theta+\hat{\boldsymbol{r}} \cdot \boldsymbol{u}_{1} \cos \theta\right) \hat{\boldsymbol{x}}+\left(\hat{\boldsymbol{\theta}} \cdot \boldsymbol{u}_{1} \cos \theta+\hat{\boldsymbol{r}} \cdot \boldsymbol{u}_{1} \sin \theta\right) \hat{\boldsymbol{y}}\right)\right|_{r=a},
$$

and integrate (2.14) to give the ICEP velocity of the near-cylinder,

$$
\boldsymbol{U}_{\mathrm{ICEP}}=-\frac{5}{8} \epsilon U_{0}[\cos (-2 \gamma) \hat{\boldsymbol{x}}+\sin (-2 \gamma) \hat{\boldsymbol{y}}] .
$$

Note that the cylinder moves in the direction $(-2 \gamma)$ : towards the blunt end when $\gamma=0$ or $\gamma=\pi$ (fore-aft asymmetric), and towards the pointed end when $\gamma= \pm \pi / 2$ (left-right asymmetric). Furthermore, using (2.15), we find that the near-cylinder has no ICEP rotation.

To solve for the flow itself, we use a streamfunction for $\boldsymbol{u}_{1}$,

$$
\psi_{1}=\frac{U_{0} a}{8} \sum_{n}\left(A_{n}^{+} \frac{a^{n}}{r^{n}}+B_{n}^{+} \frac{a^{n-2}}{r^{n-2}}\right) \sin (n \theta+2 \gamma)+\left(A_{n}^{-} \frac{a^{n}}{r^{n}}+B_{n}^{-} \frac{a^{n-2}}{r^{n-2}}\right) \sin (n \theta-2 \gamma),
$$

and find $A_{1}^{+}=-10, B_{1}^{+}=5, A_{1}^{-}=3, B_{1}^{-}=0, A_{3}^{-}=6, B_{3}^{-}=-3, A_{5}^{-}=10$, and $B_{5}^{-}=-5$, with all higher terms zero. We have deliberately excluded the Stokeslet term (proportional to $\log r$ ) from our expansion in order that (4.26) represent the ICEO flow around a freely suspended (force- and torque-free) near-cylinder. Flows for $\gamma=0$ (fore-aft asymmetric) and $\gamma=\pi / 2$ are shown in figure $5(c-d)$.

That (4.25) gives the correct ICEP velocity can be seen from the flow at infinity in (4.26), represented by the $B_{1}$ terms. Furthermore, that a solution can be obtained without $n=0$ terms (i.e. no rotation at infinity), confirms that the near-cylinder does not rotate.

Generally, perturbations that break reflectional symmetry ( $P_{n}$, where $n$ is odd) lead to translational ICEP motion, but not rotation. This can be seen from the form of the integrals (2.14)-(2.15),

$$
\begin{aligned}
U_{I C E P} & \sim \int\left(u_{1}^{\theta}, u_{1}^{r}\right) *(\sin \theta, \cos \theta) \mathrm{d} \theta, \\
\Omega_{I C E P} & \sim \int u_{1}^{\theta} \mathrm{d} \theta .
\end{aligned}
$$

A non-zero $U_{I C E P}$ requires $u_{s}$ to contain a term proportional to $\sin \theta$ or $\cos \theta$, whereas a non-zero $\Omega_{I C E P}$ requires a constant term. From (4.20)-(4.21), one can see that odd- $n$ $P_{n}$ perturbations give rise to slip velocity perturbations $u_{1}$ containing only odd- $n$ harmonic functions ( $\sin n \theta$ and $\cos n \theta$, with $n$ odd) and thus can cause translation, but not rotation. Perturbations that break rotational, but not reflectional, symmetry ( $P_{n}$, where $n$ is even) give $u_{1}$ with even- $n$ harmonic functions and lead to ICEP rotation, but not translation. Similarly, even- $n$ perturbations can be shown to rotate via DEP. 
Note that the correction to this analysis occurs at $O\left(\epsilon^{2}\right)$. Note, however, that the transformation $\epsilon \rightarrow-\epsilon$ reverses the 'direction' of the asymmetry of an ICEP swimmer - but should not affect its ICEP velocity. Thus although the flows and fields have $O\left(\epsilon^{2}\right)$ corrections, the ICEP velocities (or analogously rotations) are accurate to $O\left(\epsilon^{3}\right)$.

The analogous problem for an elongated $\left(P_{2}\right)$ near-cylinder rotates with angular velocity

$$
\Omega_{z}=\frac{9}{4} \epsilon \frac{\varepsilon E_{b}^{2}}{\eta} \sin 2 \gamma .
$$

The prefactor $9 / 4$ reflects two contributions: $3 / 2$ comes from ICEP and $3 / 4$ from DEP. Note that the elongated bodies rotate so that the long axis is oriented along the field axis.

\subsection{ICEP motion of a near-sphere}

Next, we consider the analogous three-dimensional problem of a nearly spherical conductor, with perturbed radius

$$
R=a[1+\epsilon f(\theta)] .
$$

(Note that the perturbation considered here is axisymmetric; a helically asymmetric perturbation will be discussed shortly.) A normal and two tangent vectors describe the surface,

$$
\begin{aligned}
\hat{\boldsymbol{n}} & =\hat{\boldsymbol{r}}-\epsilon f_{\theta} \hat{\boldsymbol{\theta}}+O\left(\epsilon^{2}\right), \\
\hat{\boldsymbol{t}} & =\hat{\boldsymbol{\theta}}+\epsilon f_{\theta} \hat{\boldsymbol{r}}+O\left(\epsilon^{2}\right), \\
\hat{\boldsymbol{\phi}} & =\hat{\boldsymbol{\phi}},
\end{aligned}
$$

where $f_{\theta}=\partial f / \partial \theta$ as above. As for the near-cylinder, we consider the simplest symmetry-breaking perturbation,

$$
f(\theta)=P_{3}(\cos \theta),
$$

'pointing' in the positive $\hat{\boldsymbol{x}}$-direction $(\theta=0)$. A constant electric field is directed along the angle $\gamma$ in the $(x, y)$-plane. For simplicity, we decompose the applied field into two components: an $\hat{\boldsymbol{x}}$-component $\alpha E_{b}$, and a $\hat{\boldsymbol{y}}$-component $\beta E_{b}$. The calculation is entirely analogous to the two-dimensional case described above, and thus we simply provide the main results.

\subsubsection{Electric field}

The electric field is givenby

$$
\begin{gathered}
\Phi_{0}=-E_{b}(\beta \sin \theta \cos \phi+\alpha \cos \theta)\left(r+\frac{a^{3}}{2 r^{2}}\right) \\
\Phi_{1}=\alpha E_{b} a\left[\frac{3}{28}(1+3 \cos 2 \theta)\left(\frac{a}{r}\right)^{3}-\frac{3}{224}(9+20 \cos 2 \theta+35 \cos 4 \theta)\left(\frac{a}{r}\right)^{5}\right] \\
-\beta E_{b} a \cos \phi\left[\frac{3}{14} \sin 2 \theta\left(\frac{a}{r}\right)^{3}+\frac{15}{224}(2 \sin 2 \theta+7 \sin 4 \theta)\left(\frac{a}{r}\right)^{5}\right] .
\end{gathered}
$$

Note that as with the near-cylinder described above, a $P_{3}$ perturbation does not introduce a dipole, giving no DEP torque (and, as seen below, no ICEP rotation). 
The induced zeta potential $\zeta_{i}$ is then easily obtained as well, giving

$$
\begin{gathered}
\zeta_{0}=\frac{3}{2} E_{b} a(\alpha \cos \theta+\beta \cos \phi \sin \theta), \\
\zeta_{1}=\frac{3}{224} \epsilon E_{b} a[\alpha(1-4 \cos 2 \theta+35 \cos 4 \theta)+\beta \cos \phi(26 \sin 2 \theta+35 \sin 4 \theta)] .
\end{gathered}
$$

\subsubsection{Fluid flow}

As above, we pose an expansion for the fluid velocity, $\boldsymbol{u}=\boldsymbol{u}_{0}+\epsilon \boldsymbol{u}_{1}+\ldots$ and obtain the leading-order fluid flow boundary conditions

$$
\begin{aligned}
\left.\hat{\boldsymbol{e}}_{\|} \cdot \boldsymbol{u}_{0}\right|_{a} & =\hat{\boldsymbol{e}}_{\|} \cdot \boldsymbol{u}_{0}^{s}(\theta), \\
\left.\hat{\boldsymbol{r}} \cdot \boldsymbol{u}_{0}\right|_{a} & =0,
\end{aligned}
$$

where $\hat{\boldsymbol{e}}_{\|}$is a tangent vector, either $\hat{\boldsymbol{t}}$ or $\hat{\boldsymbol{\phi}}$, and

$$
\boldsymbol{u}_{0}^{s}=\frac{9}{4} \frac{\varepsilon}{\eta} E_{b}^{2} a(\alpha \cos \theta+\beta \cos \phi \sin \theta)[-(\beta \cos \phi \cos \theta-\alpha \sin \theta) \hat{\boldsymbol{\theta}}+\beta \sin \phi \hat{\boldsymbol{\phi}}] \text {. }
$$

The leading-order ICEO flow field is that of Gamayunov et al. (1986) and Squires \& Bazant (2004):

$$
\begin{aligned}
& u_{r}=\frac{9 a^{2}\left(a^{2}-r^{2}\right)}{16 r^{4}} U_{0}(1+3 \cos 2 \bar{\theta}), \\
& u_{\bar{\theta}}=\frac{9 a^{4}}{8 r^{4}} U_{0} \sin 2 \bar{\theta} .
\end{aligned}
$$

Here, for simplicity of notation, we have used a spherical coordinate system rotated so that the polar angle $\bar{\theta}$ is measured relative to the electric field.

The boundary conditions for $\boldsymbol{u}_{1}$ are given by

$$
\begin{aligned}
\left.\hat{\boldsymbol{e}}_{\|} \cdot \boldsymbol{u}_{1}\right|_{a} & =\hat{\boldsymbol{e}}_{\|} \cdot u_{1}^{s}-a f \frac{\partial}{\partial r}\left[\hat{\boldsymbol{e}}_{\|} \cdot \boldsymbol{u}_{0}\right]_{r=a}, \\
\left.\hat{\boldsymbol{r}} \cdot \boldsymbol{u}_{1}\right|_{a} & =\left[f_{\theta} \hat{\boldsymbol{\theta}} \cdot \boldsymbol{u}_{0}-a f \frac{\partial}{\partial r}\left(\hat{\boldsymbol{r}} \cdot \boldsymbol{u}_{0}\right)\right]_{r=a},
\end{aligned}
$$

where we have used (4.31) and (4.32).

The terms $u_{1}^{s}$ are somewhat involved, but follow from (4.14) and are straightforward to obtain with a symbolic mathematics program. Using (4.43), we find

$$
a f(\theta) \partial_{r} \boldsymbol{u}_{0}^{\|}=-\left.4 f(\theta) \boldsymbol{u}_{0}^{\|}\right|_{a} .
$$

so that the right-hand side of (4.44) is known. Using (4.41), $f_{\theta} \hat{\boldsymbol{\theta}} \cdot \boldsymbol{u}_{0}$ is straightforward to compute. Calculating the partial derivative of (4.43) gives

$$
\left.\partial_{r}\left(\hat{\boldsymbol{r}} \cdot \boldsymbol{u}_{0}^{r}\right)\right|_{a}=-\frac{9}{8 a} U_{0}(1+3 \cos 2 \bar{\theta}) .
$$

To express this in the correct coordinate system, we write

$$
\left.\partial_{r}\left(\hat{\boldsymbol{r}} \cdot \boldsymbol{u}_{0}^{r}\right)\right|_{a}=-\frac{9}{8 a} U_{0}\left(1+3 \frac{\bar{x}^{2}-\bar{y}^{2}-\bar{z}^{2}}{a^{2}}\right),
$$

where the barred Cartesian coordinates are rotated an angle $\gamma$ about the $\hat{z}$-axis from the standard spherical coordinate system. Using

$$
\begin{aligned}
& \bar{x}=a(\cos \gamma \cos \theta-\sin \gamma \sin \theta \cos \phi), \\
& \bar{y}=a(\sin \gamma \cos \theta+\cos \gamma \sin \theta \cos \phi), \\
& \bar{z}=a \sin \theta \sin \phi,
\end{aligned}
$$


(a)

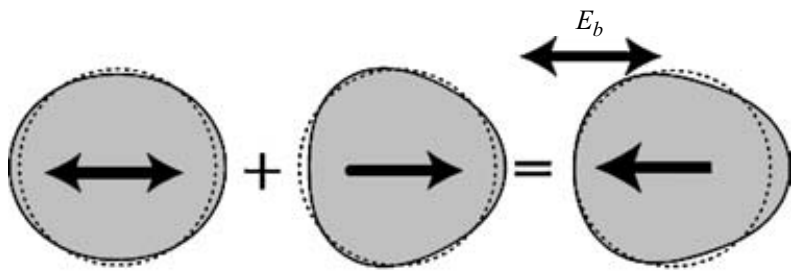

(b)

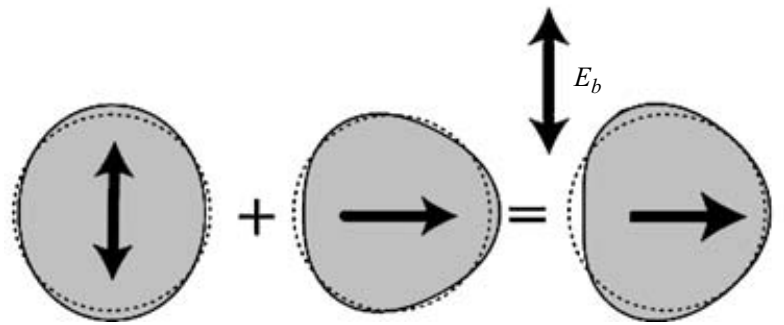

FIGURE 6. Asymmetric near-spheres can be 'designed' to translate in a particular direction relative to the applied electric field. (a) A near-sphere with both $P_{2}$ and $P_{3}$ perturbations aligned along the same axis rotates to align with the applied field, and moves along the field in the direction of its blunt end. $(b)$ A near-sphere with $P_{2}$ perturbation oriented perpendicular to a $P_{3}$ perturbation rotates so that the $P_{2}$-axis aligns with the applied field, and the near-sphere then moves perpendicular to the field in the direction of its sharp end.

in (4.48), we obtain an expression for the final term of (4.45). We then use (2.14) to evaluate the velocity, giving

$$
\boldsymbol{U}_{\text {ICEP }}=\frac{3}{28} \epsilon U_{0}[-(1+3 \cos 2 \gamma) \hat{\boldsymbol{x}}+2 \sin (2 \gamma) \hat{\boldsymbol{y}}],
$$

and using (2.15) we see there is no ICEP rotation.

As with the near-cylinder, rotations occur for an elongated near-sphere, with radius

$$
f(\theta)=P_{2}(\cos \theta) .
$$

In this case, no ICEP velocity occurs (as expected by symmetry), but the elongated near-sphere rotates with angular velocity

$$
\Omega_{z}=\frac{9}{8} \sin 2 \gamma
$$

to align itself with the field. Of the prefactor $9 / 8,81 / 80$ comes from ICEP and 9/80 from DEP.

We conclude with some general remarks about shape asymmetries and how their understanding allows metallic particles to be 'designed' to give a particualar ICEP behaviour. Although ICEP is a nonlinear phenomenon, shape-perturbation effects come in at leading order, whereas interactions between multiple shape perturbations,

$$
R=a\left(1+\sum_{n} \epsilon_{n} P_{n}(\cos \theta)\right),
$$

are of order $\epsilon_{n}^{2}$. Thus the leading-order effect of multiple shape asymmetries upon ICEP behaviour can be simply superposed. Regardless of $P_{3}$, a particle with positive $P_{2}$ perturbation rotates to align its $P_{2}$-axis with the applied field. Once aligned, however, the orientation of the $P_{3}$ component determines the ICEP swimming velocity. A nearsphere with positive $P_{2}$ and $P_{3}$ perturbations, both aligned along the same axis as in figure $6(a)$, will rotate to align with the field, then translate along field lines in the 


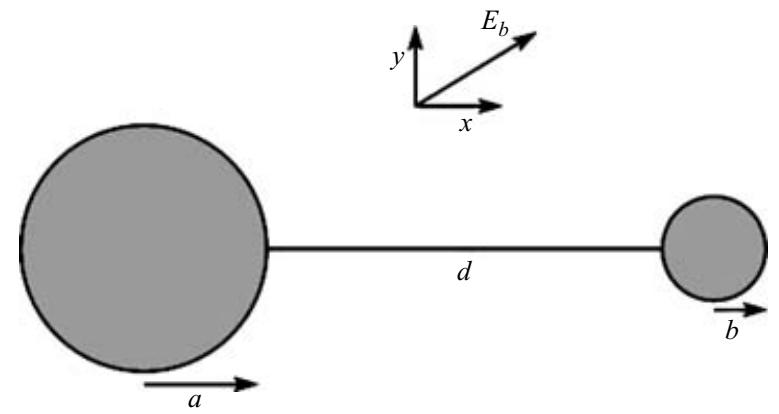

FiguRE 7. A simple asymmetric conducting body which consists of two differently sized cylinders connected by a negligibly thin conducting wire. An externally applied AC or DC electric field gives rise to an induced-charge electro-osmotic flow which causes a net electrophoretic motion.

direction of its blunt end. A near-sphere with positive $P_{2}$, and a $P_{3}$ component oriented in a perpendicular direction, as in figure $6(b)$, will swim in the plane perpendicular to the field, towards the sharp end. If the $P_{2}$ component is negative (disk-like), the particle will rotate so that the $P_{2}$-axis is perpendicular to the field. How the particle swims then depends on the orientation of the $P_{3}$ component.

\subsubsection{Helical perturbations of a sphere}

We have seen that breaking reflectional symmetry gives rise to a translational ICEP motion. Therefore, we might expect that breaking helical symmetry would give rise to a steady rotational motion. The corresponding calculation is analogous to the above calculations and thus conceptually straightforward, but is more involved computationally, as spherical harmonics are inherently non-helical. However, we can show generally that a helical near-sphere does not rotate via ICEP, at least to $O(\epsilon)$, using symmetry arguments. Since the helicity remains unchanged under an $\epsilon \rightarrow-\epsilon$ transformation, we would expect any ICEP rotation to occur in the same direction under such a transformation. However, any $O(\epsilon)$ ICEP rotation would change sign (i.e. direction) under $\epsilon \rightarrow-\epsilon$. This does not, of course, rule out helically asymmetric conductors that steadily rotate about an applied field. Rather, it restricts such rotations to significantly asymmetric bodies (for example, the composite ICEO spinners described in $\S 3.6$.)

\section{Composite bodies}

The above examples concerned bodies whose shape was symmetric or nearly symmetric. As a final example, we consider a significantly asymmetric object that can nonetheless be treated perturbatively: a composite body consisting of two symmetric conductors (radii $a>b$ ), held a distance $d$ apart but electrically connected, as in figure 7. This object is similar to the three-dimensional composite 'dumb-bells' whose (fixed-charge) electrophoretic mobilities were studied theoretically by Fair \& Anderson (1990) and Long \& Ajdari (1996), and experimentally by Fair \& Anderson (1992). Here we start with composite bodies composed of spheres rather than cylinders, so as to initially avoid the issues raised by two-dimensional Stokes flow. However, cylindrical composites would be easiest to fabricate, as they would simply involve two different-sized wires placed through a channel, and electrically connected outside the channel. 


\subsection{Two-sphere composite body}

We consider a composite body consisting of two spheres of radii $a$ and $b=\epsilon a$, located at $x=0$ and $x=d$, respectively, where the separation $d$ is large compared to the radii, and where $0<\epsilon<1$ (that is, $a>b$ ). The spheres are electrically connected, so that charge may flow freely between the two. We will employ a shorthand notation for coordinates, in which we use two different spherical coordinate systems, one centred on each sphere, and denoted by $\boldsymbol{r}_{a}$ and $\boldsymbol{r}_{b}$. We apply an electric field $\boldsymbol{E}_{b}=E_{b}(\cos \gamma \hat{\boldsymbol{x}}+\sin \gamma \hat{\boldsymbol{y}})$, and define the 'zero' of the potential $\Phi$ to occur at $\boldsymbol{r}_{a}=\mathbf{0}$. Note also that both $\theta_{a}^{\prime}$ and $\theta_{b}^{\prime}$ are zero along the axis of the electric field.

To leading order, each sphere is immersed in a constant electric field $\boldsymbol{E}_{b}$, and the zeta potential induced around each is given by

$$
\begin{aligned}
& \zeta_{a}=\zeta_{0}+E_{b} a \cos \theta_{a}^{\prime}, \\
& \zeta_{b}=\zeta_{0}+E_{b} d \cos \gamma+E_{b} b \cos \theta_{b}^{\prime},
\end{aligned}
$$

where $\theta^{\prime}$ is the angle measured relative to the axis of the electric field $\boldsymbol{E}_{b}$, and $\zeta_{0}$ enforces charge conservation (2.6), giving

$$
\zeta_{0}=-E_{b} \frac{b^{2}}{a^{2}+b^{2}} \mathrm{~d} \cos \gamma
$$

and correspondingly

$$
\begin{aligned}
& \zeta_{a}^{\prime}=-E_{b} \frac{b^{2}}{a^{2}+b^{2}} \mathrm{~d} \cos \gamma, \\
& \zeta_{b}^{\prime}=E_{b} \frac{a^{2}}{a^{2}+b^{2}} \mathrm{~d} \cos \gamma,
\end{aligned}
$$

where the prime denotes the constant (monopolar) component of the induced zeta potential.

If the spheres were free to move independently, each would move electrophoretically owing to the interaction of the field with the induced zeta potentials. The dipolar components of the zeta potentials give no motion, and the monopolar components would give an electrophoretic velocity

$$
\begin{aligned}
\boldsymbol{U}_{a}^{f} & =-\frac{\varepsilon E_{b}^{2} d}{\eta} \frac{b^{2}}{a^{2}+b^{2}}(\cos \gamma \hat{\boldsymbol{x}}+\sin \gamma \hat{\boldsymbol{y}}) \cos \gamma, \\
\boldsymbol{U}_{b}^{f} & =\frac{\varepsilon E_{b}^{2} d}{\eta} \frac{a^{2}}{a^{2}+b^{2}}(\cos \gamma \hat{\boldsymbol{x}}+\sin \gamma \hat{\boldsymbol{y}}) \cos \gamma .
\end{aligned}
$$

However, the spheres are not free to float independently. Equal and opposite forces $\pm F \hat{\boldsymbol{x}}$ keep them from moving relative to each other,

$$
\hat{\boldsymbol{x}} \cdot \boldsymbol{U}_{a}^{f} \cos \gamma+\frac{F}{6 \pi \eta a}=\hat{\boldsymbol{x}} \cdot \boldsymbol{U}_{b}^{f} \cos \gamma-\frac{F}{6 \pi \eta b},
$$

giving

$$
\frac{F}{6 \pi \eta}=\frac{a b}{a+b} \frac{\varepsilon E_{b}^{2} d}{\eta} \cos ^{2} \gamma .
$$

The velocity of each sphere is thus given by

$$
\boldsymbol{U}_{a}=U_{0} \frac{a b(a-b)}{(a+b)\left(a^{2}+b^{2}\right)} \cos ^{2} \gamma \hat{\boldsymbol{x}}-U_{0} \frac{b^{2}}{a^{2}+b^{2}} \sin \gamma \cos \gamma \hat{\boldsymbol{y}},
$$




$$
\boldsymbol{U}_{b}=U_{0} \frac{a b(a-b)}{(a+b)\left(a^{2}+b^{2}\right)} \cos ^{2} \gamma \hat{\boldsymbol{x}}+U_{0} \frac{a^{2}}{a^{2}+b^{2}} \sin \gamma \cos \gamma \hat{\boldsymbol{y}},
$$

where $U_{0}=\left(\varepsilon E_{b}^{2} d / \eta\right)$. The first term in each expression represents a uniform translation along the axis of the composite body, in the direction of the smaller particle. The second term in each represents motion perpendicular to the body axis, giving both translation

$$
U_{y}=\frac{U_{0}}{4} \frac{a^{2}-b^{2}}{a^{2}+b^{2}} \sin 2 \gamma,
$$

perpendicular to the field, and rotation

$$
\Omega_{z}=\frac{\varepsilon E_{b}^{2}}{2 \eta} \sin 2 \gamma
$$

that tends to align the body with the field.

Finally, we note that the ICEP velocity of the composite two-sphere body is greatest when

$$
\left.\frac{a}{b}\right|_{\max }=\frac{1+\sqrt{5}}{2}-\sqrt{\frac{1+\sqrt{5}}{2}} \approx 0.35,
$$

and remind the reader that these results hold in the limit where the spheres are well separated $(d \gg a, b)$.

\subsection{Composite cylinders}

A composite body composed of cylinders is perhaps the easiest asymmetric body to fabricate, as one can simply insert two different-sized wires through a channel, and electrically connect them outside of the channel. The analysis is similar to that above, giving induced zeta potentials with constant components

$$
\begin{aligned}
\zeta_{a}^{\prime} & =-E_{b} \frac{b}{a+b} d \cos \gamma, \\
\zeta_{b}^{\prime} & =E_{b} \frac{a}{a+b} d \cos \gamma .
\end{aligned}
$$

The ICEP velocity of each cylinder, if it were freely floating, would then be

$$
\begin{aligned}
U_{a}^{f} & =U_{0} \frac{b}{a+b}\left(-\cos ^{2} \gamma \hat{\boldsymbol{x}}-\sin \gamma \cos \gamma \hat{\boldsymbol{y}}\right), \\
U_{b}^{f} & =U_{0} \frac{a}{a+b}\left(\cos ^{2} \gamma \hat{\boldsymbol{x}}+\sin \gamma \cos \gamma \hat{\boldsymbol{y}}\right) .
\end{aligned}
$$

As above, equal and opposite forces $\pm F \hat{\boldsymbol{x}}$ keep the cylinders from moving relative to each other. Although forced motion is ill-defined in two-dimensional Stokes flow, the motion of two cylinders subject to equal and opposite forces is not, giving leading-order velocities

$$
\begin{aligned}
U_{a} & =\frac{F}{8 \pi \mu}(2 \ln d / a-1), \\
U_{b} & =-\frac{F}{8 \pi \mu}(2 \ln d / b-1) .
\end{aligned}
$$


Thus, the component of the ICEP velocity aligned with the axis of the composite body is

$$
U_{x}=U_{0}\left(\frac{2 \ln d / a-1}{2\left(\ln d^{2} / a b-1\right)}-\frac{b}{a+b}\right) \cos ^{2} \gamma .
$$

Furthermore, the ICEP velocity perpendicular to the body axis is

$$
U_{y}=U_{0} \frac{a-b}{a+b} \frac{\sin 2 \gamma}{2},
$$

and the body rotates with angular velocity

$$
\Omega_{z}=\frac{\varepsilon E_{b}^{2}}{\eta} \sin 2 \gamma,
$$

to align with the applied field.

\section{Induced-charge electrophoresis in a uniform gradient field}

The preceding examples have all involved ICEO in systems whose asymmetry lies in the geometry of the polarizable surface. In this section, we consider systems whose broken symmetry occurs via a non-uniform applied electric field,

$$
\boldsymbol{E}_{a}=\boldsymbol{E}_{b}+\boldsymbol{G}_{b} \cdot \boldsymbol{r},
$$

where the (spatially constant) $\boldsymbol{G}_{b}$ gives a gradient in the field intensity (or electrostatic energy $\left.\varepsilon \boldsymbol{E}^{2}\right)$,

$$
\nabla\left|\boldsymbol{E}_{a}\right|^{2}=2 \boldsymbol{E}_{b} \cdot \boldsymbol{G}_{b} .
$$

(Hereinafter, we will drop the subscripts.) We will demonstrate that a symmetric conducting object in an AC field experiences an induced-charge electrophoretic motion that drives it up the field gradient, and a dielectrophoretic force that drives it down the field gradient, consistent with the results of Shilov \& Simonova (1981) and Simonova et al. (2001) for spheres. The net velocity, however, is geometry-dependent.

\subsection{Conducting sphere in uniform-gradient field}

We begin by examining the motion of an ideally polarizable sphere of radius $a$ in the applied electric field (6.1) with a uniform gradient. Although this example has been analysed by Shilov \& Simonova (1981), let us study it briefly within the framework we have built here; we will then treat the cylindrical case to highlight the crucial role played by geometry, which it seems has not previously been explored.

The steady-state electric potential is given by

$$
\Phi=-E_{i} r_{i}-\frac{a^{3}}{2} \frac{E_{i} r_{i}}{r^{3}}-\frac{1}{2} G_{i j} r_{i} r_{j}-\frac{a^{5}}{9} G_{i j}\left(-\frac{\delta_{i j}}{r^{3}}+\frac{3 r_{i} r_{j}}{r^{5}}\right),
$$

and the zeta potential is then given by

$$
\zeta=\frac{3 a}{2} E_{i} \hat{\boldsymbol{r}}_{i}+\frac{5 a^{2}}{6} G_{i j} \hat{\boldsymbol{r}}_{i} \hat{\boldsymbol{r}}_{j}
$$

Note that the charge-conservation equation (2.6), is satisfied naturally, since $G_{i j}$ is traceless and $G_{i j} \int \hat{\boldsymbol{r}}_{i} \hat{\boldsymbol{r}}_{j} \mathrm{~d} \Omega=0$. The tangential field outside the double layer is given by

$$
E_{k}(a)=\frac{3}{2} E_{k}-\frac{3}{2} E_{j} \hat{r}_{j} \hat{r}_{k}+\frac{5 a}{3} G_{j k} \hat{r}_{j}-\frac{5 a}{3} G_{i j} \hat{r}_{i} \hat{r}_{j} \hat{r}_{k},
$$


where $\hat{\boldsymbol{r}}=\boldsymbol{r} / \boldsymbol{r}$. The local ICEO slip velocity is given by (1.1), using (6.4) and (6.5). Using (2.12), the ICEP velocity of the sphere is given by

$$
U_{k}=\frac{\varepsilon}{\eta} \frac{1}{4 \pi} \int\left(\frac{3 a}{2} E_{i} \hat{r}_{i}+\frac{5 a^{2}}{6} G_{i j} \hat{r}_{i} \hat{r}_{j}\right)\left(\frac{3}{2} E_{k}-\frac{3}{2} E_{j} \hat{r}_{j} \hat{r}_{k}+\frac{5 a}{3} G_{j k} \hat{r}_{j}-\frac{5 a}{3} G_{i j} \hat{r}_{i} \hat{r}_{j} \hat{r}_{k}\right) \mathrm{d} \Omega .
$$

Of these, only three terms are non-zero:

$$
U_{k}=\frac{\varepsilon}{\eta} \frac{1}{4 \pi} \int\left(\frac{5 a^{2}}{2} G_{j k} E_{i} \hat{r}_{i} \hat{r}_{j}-\frac{5 a^{2}}{2} G_{i j} E_{l} \hat{r}_{i} \hat{r}_{i} \hat{r}_{j} \hat{r}_{k}-\frac{5 a^{2}}{4} G_{i j} E_{l} \hat{r}_{i} \hat{r}_{j} \hat{r}_{k} \hat{r}_{l}\right) \mathrm{d} \Omega .
$$

The first two terms,

$$
U_{k}=\frac{\varepsilon}{\eta} \frac{1}{4 \pi} \int\left(\frac{5 a^{2}}{2} G_{j k} E_{i} \hat{r}_{i} \hat{r}_{j}-\frac{5 a^{2}}{2} G_{i j} E_{l} \hat{r}_{i} \hat{r}_{i} \hat{r}_{j} \hat{r}_{k}\right) \mathrm{d} \Omega=\frac{\varepsilon}{2 \eta} a^{2} G_{i k} E_{i},
$$

give a motion up the gradient that results when the gradient field drives the (dipolar) charge cloud that has been induced by the constant component of the field. The third term,

$$
U_{k}=-\frac{\varepsilon}{\eta} \frac{1}{4 \pi} \int \frac{5 a^{2}}{4} G_{i j} E_{l} \hat{r}_{i} \hat{r}_{j} \hat{r}_{k} \hat{r}_{l} \mathrm{~d} \Omega=-\frac{\varepsilon}{6 \eta} a^{2} G_{i k} E_{i}
$$

causes motion down the gradient, and results when the constant field drives the (quadrupolar) charge cloud that has been induced by the gradient in the field. The resulting velocity is

$$
\boldsymbol{U}=\frac{\varepsilon}{\eta} \frac{a^{2}}{3} \boldsymbol{G} \cdot \boldsymbol{E} \equiv \frac{\varepsilon}{\eta} \frac{a^{2}}{6} \nabla\left|\boldsymbol{E}_{a}^{2}\right|
$$

so that a conducting sphere experiences an ICEP velocity up the field strength gradient.

The ICEP motion up the gradient is counteracted by dielectrophoretic motion. From (6.3), the induced dipole is $\boldsymbol{d}=-2 \pi \varepsilon \boldsymbol{E} \boldsymbol{a}^{3}$, which interacts with the gradient field according to (2.22) to give a DEP force

$$
\boldsymbol{F}_{\mathrm{DEP}}=-\pi \varepsilon a^{3} \nabla\left|\boldsymbol{E}_{a}^{2}\right|
$$

which causes the sphere to move with Stokes velocity

$$
\boldsymbol{U}_{\mathrm{DEP}}=-\frac{\varepsilon}{\eta} \frac{a^{2}}{6} \nabla\left|\boldsymbol{E}_{a}^{2}\right| .
$$

Remarkably, the dielectrophoretic motion (equation (6.12)) has an identical magnitude, but opposite direction, to the ICEP velocity (equation (6.10)). Thus no motion results, as was originally demonstrated by Shilov \& Simonova (1981). However, it is significant to note that the flow fields established by each of these two physical effects differ significantly: the DEP motion is force-driven and establishes a flow that decays with distance as $r^{-1}$. The ICEP motion, on the other hand, is force-free and decays as $r^{-2}$. Thus although a metallic sphere does not move in a field gradient, it does establish a persistent long-ranged fluid flow, as occurs generically when forced- and force-free motions are superposed (Squires 2001).

\subsection{Conducting cylinder in uniform-gradient field}

Another significant point to note is that the precise cancellation of DEP and ICEP velocities seen above is not universal, but geometry-dependent. The clearest demonstration of this fact follows from the two-dimensional (cylindrical) analogue of the 
above problem, which to our knowledge has not been studied before. A conducting cylinder climbs gradients due to ICEP, which is force-free and well-defined. Dielectrophoresis, on the other hand, exerts a force on the cylinder, whose resulting twodimensional Stokes flow is ill-defined. Thus the DEP motion of a cylinder depends sensitively on the geometry of the entire system, and differs from the ICEP velocity.

Since the cylindrical problem is entirely analogous to the spherical problem detailed above, we simply state key results (the same results are also derived in the next section using complex variables, where a general non-uniform applied field poses no more difficulty). The steady-state electrostatic potential is given by

$$
\Phi=-E_{k} r_{k}-a^{2} \frac{E_{k} r_{k}}{r^{2}}-\frac{1}{2} G_{i k} r_{i} r_{k}-\frac{a^{4}}{4} G_{i j}\left(-\frac{\delta_{i j}}{r^{2}}+\frac{2 r_{i} r_{j}}{r^{4}}\right),
$$

from which the induced zeta potential can be found to be

$$
\zeta_{i}=-\Phi(a)=2 a E_{k} \hat{r}_{k}+a^{2} G_{k j} \hat{r}_{k} \hat{r}_{j} .
$$

The parallel field adjacent to the screening cloud is given by

$$
E_{k}(a)=2 E_{k}-2 E_{j} \hat{r}_{j} \hat{r}_{k}+2 a G_{j k} \hat{r}_{j}-2 a G_{i j} \hat{r}_{i} \hat{r}_{j} \hat{r}_{k},
$$

and the net ICEP velocity,

$$
\boldsymbol{U}=\frac{\varepsilon}{\eta} \frac{a^{2}}{4} \nabla\left|\boldsymbol{E}_{a}\right|^{2},
$$

then follows. The cylinder, if free to move, climbs the field strength gradient via ICEP. Conversely, a cylinder that is held in place would pump the fluid down the field strength gradient.

The DEP force follows from the interaction between the induced dipole moment $\left(\boldsymbol{p}=-2 \pi \varepsilon a^{2} \boldsymbol{E}\right)$ and the gradient field via $\boldsymbol{F}=\boldsymbol{p} \cdot \nabla \boldsymbol{E} \equiv \boldsymbol{G}_{b} \cdot \boldsymbol{p}$ to give a DEP force per unit length

$$
\boldsymbol{F}_{\mathrm{DEP}}=-\pi \varepsilon a^{2} \nabla\left|\boldsymbol{E}_{a}\right|^{2},
$$

down the field gradient. Since, however, two-dimensional forced Stokes flow is divergent and ill-defined, no DEP velocity results unless some length scale can regularize the flow at large distances - whether set by inertia, the cylinder length, or the nearest wall.

However, a cylinder whose position is fixed, and which is subjected to a gradient field, will pump fluid down the gradient. Holding the cylinder in place requires a force to balance DEP (which leads to no flow), as well as a force to counteract the ICEP motion, which gives rise to Stokeslet flow (in addition to the ICEO slip velocity), both directed down the field gradient.

\section{General non-uniform fields and shapes in two dimensions}

\subsection{Conducting cylinder in an arbitrary applied potential}

Let the complex plane, $z=x+\mathrm{i} y$, represent the coordinates transverse to a conducting cylinder, where the electrolyte occupies the region $|z|>a$. Let $\Psi(z)$ be the complex potential, i.e. $\Phi=\operatorname{Re} \Psi$, and $E=-\overline{\Psi^{\prime}}$, the electric field (a vector represented by a complex scalar). Consider an arbitrary applied potential, in the absence of the cylinder, defined by its Taylor series (valid everywhere):

$$
\Psi_{a}=\sum_{n=0}^{\infty} A_{n}(z / a)^{n},
$$


where $A_{n}$ are (complex) multipole coefficients. The first is the (real) background potential, $\Phi_{b}=A_{0}$, relative to an electrode in the external circuit (to allow for fixedpotential ICEO). The next coefficients are related to the applied electric field, $E_{a}=E_{b}+$ $G_{b} z+H_{b} z^{2} / 2+\ldots$, analogous to (6.1). The background field is $E_{b}=-\overline{A_{1}} / a$, and the background gradient, $G_{b}=-2 \overline{A_{2}} / a^{2}$. Both are related to the background fieldintensity gradient,

$$
\nabla|E|_{b}^{2}=\nabla\left|\Psi^{\prime}\right|^{2}(0)=2 \Psi^{\prime}(0) \overline{\Psi^{\prime \prime}}(0)=A_{1} \overline{A_{2}} / a^{3}=2 \overline{E_{b}} G_{b},
$$

as in (6.2). (See Bazant (2004) for similar manipulations with the complex gradient operator, $\nabla=\partial / \partial x+\mathrm{i} \partial / \partial y=2 \partial / \partial \bar{z}$.)

After double-layer charging, the complex potential in the bulk electrolyte satisfies the insulating boundary condition, $\operatorname{Im} \Psi=0$ for $|z|=a$ with $\Psi \sim \Psi_{a}$ for $|z| \rightarrow \infty$. The solution is

$$
\Psi=A_{0}+\sum_{n=1}^{\infty}\left[A_{n}(z / a)^{n}+\overline{A_{n}}(a / z)^{n}\right] \text { for }|z|>a,
$$

where the last terms are the induced multipoles on the cylinder. For example, (up to numerical prefactors) $\overline{A_{1}}$ is the dipole moment induced by the uniform field $A_{1}$ (a dipole at $\infty) ; \overline{A_{2}}$ is the quadrupole moment induced by the gradient field $A_{2}$; etc.

The conductor's potential, $\Phi_{0}$, relative to the same zero as $\Phi_{b}$, is either set externally or determined by a fixed total charge, $Q_{0}$ (per unit length), as described above. The non-uniform zeta potential along the surface, $z=a \mathrm{e}^{\mathrm{i} \theta}$, is given by

$$
\zeta(z)=\Phi_{0}-\Phi(z)=\zeta_{0}-\sum_{n=1}^{\infty}\left(A_{n} \mathrm{e}^{\mathrm{i} n \theta}+\overline{A_{n}} \mathrm{e}^{-\mathrm{i} n \theta}\right),
$$

since $\Phi=\operatorname{Re} \Psi=\Psi$ on $|z|=a$ and where $\zeta_{0}=\Phi_{0}-\Phi_{b}$ is the surface-averaged zeta potential. Assuming a linear double-layer capacitance, this is proportional to the total charge on the object (per unit length), $Q_{0}=2 \pi a C \zeta_{0}$.

The ICEO slip velocity is given by

$$
u_{s}=(\varepsilon / \eta)\left(\Phi_{0}-\Psi\right) \overline{\Psi^{\prime}} \text { for }|z|=a,
$$

and the tangential component at $z=a \mathrm{e}^{\mathrm{i} \theta}$ by

$$
u_{\theta}=\operatorname{Re}\left(\overline{i z} u_{s}\right)=a^{2} \operatorname{Im}\left(u_{s} / z\right)
$$

Substituting (7.3) yields a Fourier series for the slip velocity, from which the twodimensional Stokes flow is straightforward to calculate, e.g. using the streamfunction (4.26). Some examples are given in figure 8.

In two dimensions, the Stone-Samuels formula for the ICEP velocity can be recast as a contour integral,

$$
U_{I C E P}=\frac{\varepsilon}{2 \pi \eta} \oint_{|z|=a}\left(\Psi-\Phi_{0}\right) \overline{\Psi^{\prime}} \frac{\mathrm{d} z}{\mathrm{i} z} .
$$

Although the integrand is not analytic, it is easily made so on the circle, $|z|=a$, by the substitution $\bar{z} / a=a / z$. The ICEP velocity then follows by residue calculus,

$$
U_{\text {ICEP }}=\frac{\varepsilon}{\eta a}\left(-\Phi_{0} \overline{A_{1}}+\sum_{n=1}^{\infty} A_{n-1} \overline{A_{n}}\right) .
$$


(a)

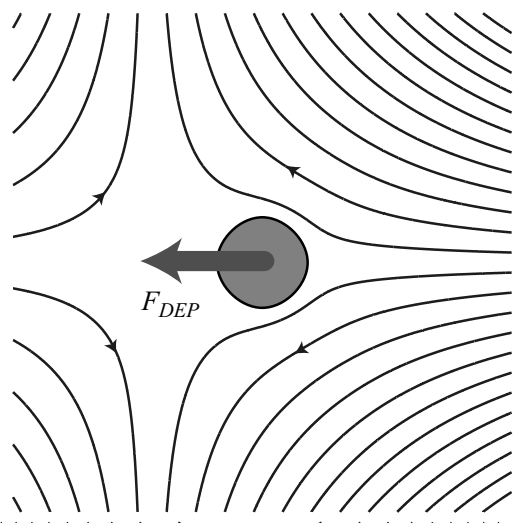

(c)

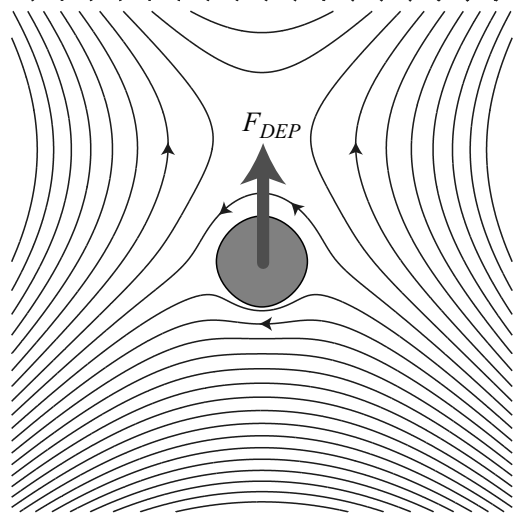

(e)

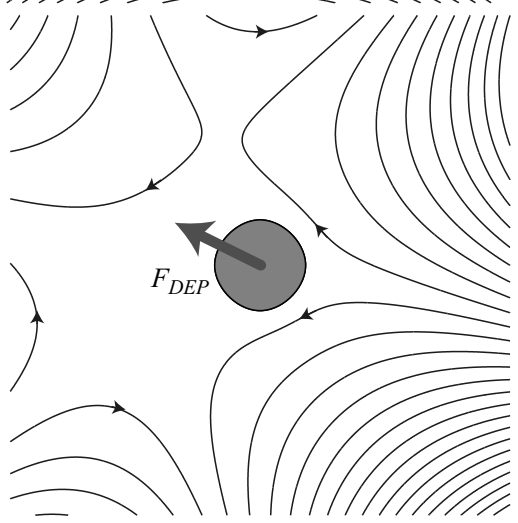

(b)

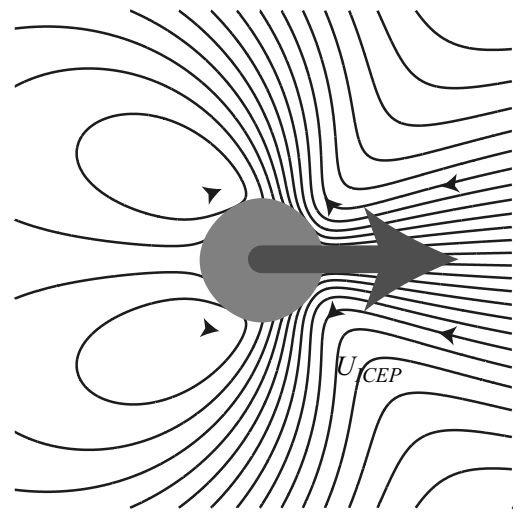

$(d)$

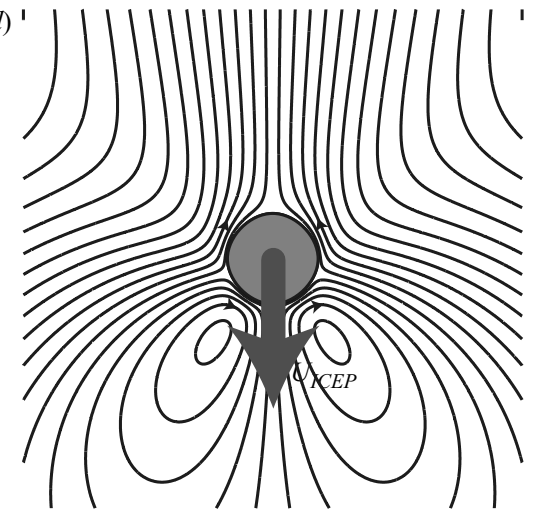

$(f)$

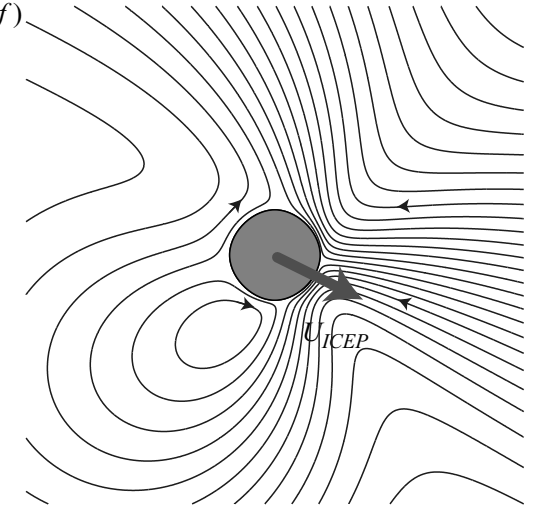

FIGURE 8. Electric fields $(a, c, e)$ and ICEO flows $(b, d, f)$ around conducting cylinders in inhomogeneous fields. ICEP velocities and DEP forces are indicated. $(a-d)$ A cylinder in linear field gradients, with $(a, b) A_{1}=1, A_{2}=0.2$, and $(c, d) A_{1}=1, A_{2}=0.2 i$. $(e, f)$ A cylinder in a quadratic field gradient, with $A_{1}=1, A_{2}=0.2+0.1 \mathrm{i}, A_{3}=0.025(1+\mathrm{i})$.

A similar calculation shows that the ICEP angular velocity vanishes,

$$
\Omega_{I C E P}=-\frac{1}{2 \pi a} \int_{0}^{2 \pi} \operatorname{Im}\left(\mathrm{e}^{-\mathrm{i} \theta} u\right) \mathrm{d} \theta=0,
$$

as it must by rotational symmetry. 
Using the relations above, the first two terms in the ICEP velocity can be recast in a more familiar form,

$$
U_{I C E P}=\frac{\varepsilon \zeta_{0} E_{b}}{\eta}+\frac{\varepsilon a^{2}}{4 \eta} \nabla|E|_{b}^{2}+\frac{\varepsilon}{\eta a} \sum_{n=3}^{\infty} A_{n-1} \overline{A_{n}} .
$$

Note that each ICEO term is quadratic in the overall magnitude of the applied potential. The first term is the normal electrophoretic velocity due to the background field acting on the total charge (which is induced by the field in fixed-potential ICEO); the second, which agrees with (6.16), results from the background field gradient acting on the induced dipole; the next, new term involves the gradient of the field gradient acting on the induced quadrupole; etc.

We now demonstrate the remarkable fact that each of these multipolar force-free ICEP motions is opposed by a forced DEP motion of the same form. The force may be calculated from the normal component of the Maxwell stress tensor (2.20),

$$
(2 / \varepsilon) \boldsymbol{\sigma}_{M} \cdot \hat{\boldsymbol{r}}=-|\boldsymbol{E}|^{2} \hat{\boldsymbol{r}}+2 \boldsymbol{E}(\boldsymbol{E} \cdot \hat{\boldsymbol{r}})=-|E|^{2} \mathrm{e}^{\mathrm{i} \theta}+2 E \operatorname{Re}\left(\mathrm{e}^{-\mathrm{i} \theta} E\right)=\mathrm{e}^{\mathrm{i} \theta} E^{2},
$$

integrating (2.18) around the cylinder,

$$
F=\frac{\varepsilon}{2 \mathrm{i}} \oint_{|z|=a}\left(\overline{\Psi^{\prime}} a / z\right)^{2} \mathrm{~d} z
$$

Substituting (7.3) and evaluating the integral by residue calculus yields the desired result,

$$
F=-(2 \pi \varepsilon / a) \sum_{n=2}^{\infty} n(n-1) A_{n-1} \overline{A_{n}} .
$$

Using (7.2), we recognize the first term as the DEP force in a uniform-gradient field,

$$
F_{D E P}=-(4 \pi \varepsilon / a) A_{1} \overline{A_{2}}=-\pi \varepsilon a^{2} \nabla|E|_{b}^{2},
$$

but equation (7.13) also contains all higher-order multipolar couplings, $A_{n-1} \overline{A_{n}}$, for any non-uniform applied field. Note that the series expansion for the ICEP velocity (7.8) has precisely the same form as that for the expansion for the electrostatic force on the object (7.13), only with coefficients of opposite sign and different magnitudes. The resulting competition between opposing force-free and forced motions explains why the electrically induced motion of polarizable colloids is so subtle.

\subsection{Conducting cylinders of arbitrary cross-section}

By applying conformal mapping to the preceding results, the ICEO slip distribution can generally be calculated for any (simply connected) two-dimensional object, in an arbitrary applied electric field. Let $w=f(z)$ be a univalent (conformal and one-toone) mapping from the fluid exterior of the object to the fluid exterior of the disk discussed above, $|w|>a$. Without loss of generality, we choose $f^{\prime}(\infty)=1$, in order to preserve the applied potential (7.1). The complex potential is obtained by simply replacing $z$ with $f(z)$ in (7.3).

The zeta potential on the surface, $|f(z)|=a$, is then given by

$$
\zeta=\zeta_{0}-\sum_{n=1}^{\infty}\left[A_{n}(z / a)^{n}+\overline{A_{n}}(a / z)^{n}\right]
$$



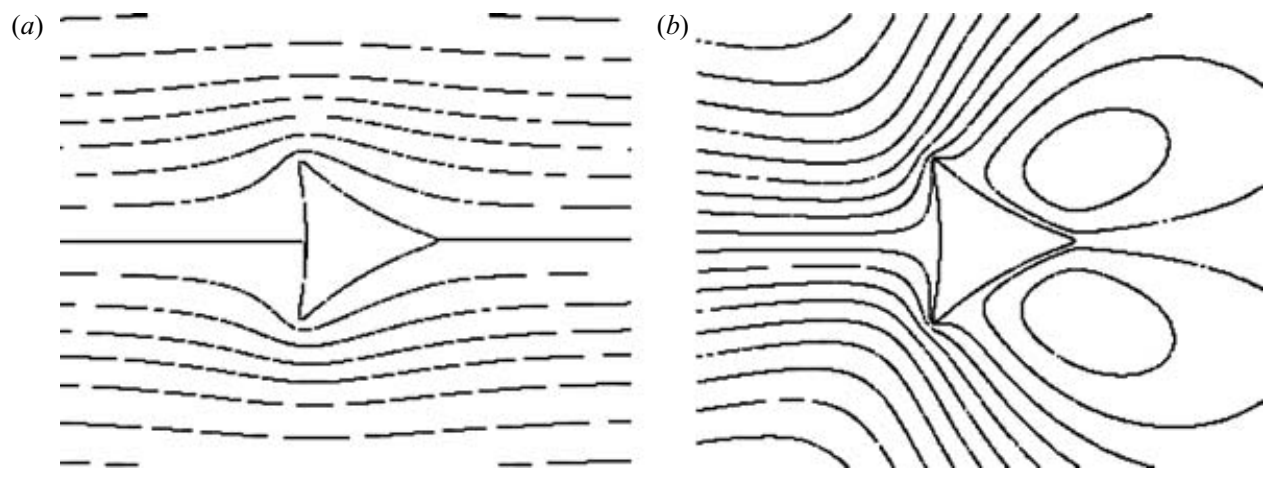

FIGURE 9. The two-dimensional electric field $(a)$ and steady ICEO flow $(b)$ around a highly asymmetric triangle-like object in a uniform background field. If fixed, the object pumps fluid from left to right by ICEO; if free to move, it swims from right to left by ICEP. Although the electric field and ICEO slip velocity for an infinite system are given exactly by our analysis, the Stokes flow in $(b)$ is calculated numerically for a large finite box (100 times larger than the object in each direction) using the finite-element package FEMLAB. (Courtesy of Yuxing Ben.)

and the electric field throughout the fluid, $|f(z)| \geqslant a$, by

$$
E=-\left(f^{\prime}(z) / a\right) \sum_{n=1}^{\infty} n\left(A_{n}(f(z) / a)^{n-1}-\overline{A_{n}}(a / f(z))^{n+1}\right) .
$$

Substituting the expressions in (7.5) yields the ICEO slip velocity on $|f(z)|=a$. Unfortunately, the Stokes flow is more complicated, and the simple Stone-Samuels formulae (2.14)-(2.15) no longer apply. Further analytical progress may be possible by exploiting analytic properties of the biharmonic streamfunction, but it is beyond the scope of this paper.

For now, we have a partial solution to the general problem, which gives the electric field and the ICEO slip velocity and leaves only the flow profile to be calculated numerically. For example, consider a 'rounded triangle' produced by the univalent map, $z=f^{-1}(w)=w-\alpha a^{3} / w^{2}$, which loses conformality with the formation of three cusps in the limit $|\alpha| \rightarrow 0.5$. As shown in figure 9 for the nearly singular case $\alpha=0.4$ (and $a=1$ ), the electric field and ICEO flow are qualitatively similar to what we calculated above for a near-cylinder of the same three-fold symmetry in figure $5(a, b)$; as before, the fluid is pumped past a pair of counter-rotating vortices from left to right, in the frame of the object, and the ICEP velocity clearly increases with the strength of the asymmetry. This comparison suggests that our perturbation analysis above may yield useful predictions, even for highly asymmetric objects.

\section{Conclusion}

In this paper, we have explored the influence of breaking various symmetries in induced-charge electro-osmotic and electrophoretic systems. The central theme of this work is that breaking spatial symmetry in any of a number of ways generically leads to an ICEO 'pumping' flow with a net directionality or, equivalently, a non-zero ICEP velocity, and can furthermore lead to a net rotation towards a steady orientation of freely suspended polarizable bodies. We have specifically considered five model asymmetric systems, each of which embodies a different aspect of generically asymmetric bodies in a manner that remains analytically tractable: (i) symmetrically shaped conductors 
with inhomogeneous surface properties; (ii) conductors with slightly asymmetric shapes; (iii) composite bodies composed of different-sized symmetric shapes (and thus potentially highly asymmetric); (iv) uniform field-strength gradients applied to symmetric shapes (with induced dipole moments); and (v) the general problem in two dimensions of asymmetric shapes in non-uniform fields (with higher-order induced multipole moments). In the cases with non-uniform applied fields, we have also calculated electrostatic forces and torques, which produce dielectrophoresis and electrorotation, in addition to the force-free slip-driven ICEP translation and rotation.

Throughout the paper, we have mentioned possible applications of ICEO and ICEP phenemena involving broken symmetries, in microfluidics and colloids, respectively, so we conclude by briefly discussing some directions for further development of the theory. Our calculations are based on the standard theory of what we call 'ICEO' flows in weak applied fields with thin double layers, also employed in the Russian literature on metal colloids (Gamayunov et al. 1986; Murtsovkin 1996), the recent work on AC electro-osmosis (Ramos et al. 1999; Ajdari 2000; Gonzalez et al. 2000), and our previous work on ICEO in microfluidics (Bazant \& Squires 2004; Squires \& Bazant 2004). In contrast, experiments on ICEO flows in polarizable colloids (Gamayunov \& Murtsovkin 1987; Murtsovkin \& Mantrov 1990), at microelectrodes in pairs (Ramos et al. 1999, 2003; Green et al. 2002) and periodic arrays (Brown et al. 2001; Mpholo et al. 2003; Studer et al. 2004), and metal structures in microchannels (Levitan et al. 2005), are usually performed at much larger voltages, in order to achieve stronger ICEO flows. Although most experiments confirm the basic scale of the flow (1.2), the observed velocities are systematically smaller than predicted by the simple model, which contains no adjustable parameters.

To some extent, it may be possible to fit the experiments by solving the classical electrokinetic equations (i.e. the Poisson-Nernst-Planck equations for ion transport coupled to the Stokes equations with an electrostatic body force for the fluid flow) in the regime of large Dukhin number. As mentioned in $\S 2$, this occurs when the total (equilibrium + induced) zeta potential, $\zeta=\zeta_{0}+\zeta_{i}$, exceeds $2 k T / e \approx 50 \mathrm{mV}$, where $\zeta_{i} \approx E_{b} a$, and generally tends to reduce ICEO flow. The case of highly charged particles, $e \zeta_{0} / 2 k T \gg 1$, subject to weak applied fields, $e \zeta_{i} / 2 k T \ll 1$, has been studied extensively in the case of normal (fixed-charge) electrophoresis (Dukhin \& Shilov 1974; Dukhin 1993), as well as ICEO around charged polarizable spheres (Murtsovkin 1996). In this regime, the equations may be linearized to describe weak concentration polarization and surface conduction in a (uniformly) highly charged double layer. Unfortunately, the case of large applied fields, $e \zeta_{i} / 2 k T \gg 1$, is more difficult to analyse, especially for uncharged particles, $\zeta_{0}=0$, owing to highly non-uniform polarization; the electrochemical problem for spheres and cylinders has been analysed by Chu (2005) using boundary-layer techniques to describe surface conduction, neutral salt adsorption and bulk diffusion (Bazant et al. 2004), but the effect of concentration polarization and diffusio-osmosis on ICEO flow at large voltages (and arbitrary Péclet number) remains an open question.

Experiments also suggest that theory of ICEO must account better for interfacial chemistry. Electrochemical interfaces, such as gold/potassium chloride, can display complicated impedance spectra, sometimes approximated by a 'constant-phase-angle' impedance $Z \propto(\mathrm{i} \omega)^{\beta}$ with $\beta$ in the range 0.6-0.9. Although controversial, this boundary condition seems to improve the fit of some experimental data (Green et al. 2002; Levitan et al. 2005). Another puzzling observation is that the strength of ICEO flow depends sensitively on the type of ions in the electrolyte (e.g. sodium chloride vs. potassium chloride) and the material of the working conductor (e.g. platinum $v$ s. gold) 
(Levitan 2005). It is also strongly suppressed with increasing concentration beyond the millimolar range, with a complicated voltage dependence (Studer et al. 2004). Studies by Oleson, Bruus \& Ajdari (2006) indicate that such complicated behaviour is not captured by such simple treatments, nor by various extensions of the theory mentioned above. In spite of these subtle issues, however, the simple model used here is able to predict qualitative (and sometimes quantitative) features of ICEO flows, while remaining analytically tractable.

\section{Appendix. Force-free motion by surface slip in two dimensions}

Brenner (1964) pioneered the use of the reciprocal theorem for Stokes flows to derive general relations between the forced and force-free (slip-driven) motion of an isolated three-dimensional body, which have been applied to ICEP by Yariv (2005). In our work, we make extensive use of the simple explicit formulae (2.12)-(2.13) of Stone \& Samuel (1996) for the translational and rotational velocities of a sphere (intended to model a swimming micro-organism) which is subject to no external force and specified slip velocity on its surface. Here we show that their results hold for two-dimensional bodies as well, despite subtleties of two-dimensional Stokes flows.

The reciprocal theorem holds that

$$
\int \boldsymbol{u} \cdot \hat{\boldsymbol{\sigma}} \cdot \hat{\boldsymbol{n}} \mathrm{d} A=\int \hat{\boldsymbol{u}} \cdot \boldsymbol{\sigma} \cdot \hat{\boldsymbol{n}} \mathrm{d} A,
$$

where the integration is taken around the boundaries, and $\hat{\boldsymbol{n}}$ is the inner normal. Here $\boldsymbol{u}$ and $\boldsymbol{\sigma}$ refer to the problem of interest: a force-free object with a specified slip velocity $\boldsymbol{u}_{s}$ and (unknown) swimming velocity $\boldsymbol{U}_{0}$ on the surface $\Gamma$. Hatted variables refer to the same body translating at velocity $\hat{U}$. Note that two-dimensional Stokes flows around forced bodies diverge logarithmically at infinity (Proudman \& Pearson 1957), and force-free flows decay as $r^{-1}$ or faster. We need not demand, however, that the hatted flow be physically reasonable - only that it solve the Stokes equations and therefore serve its role in the reciprocal theorem.

The integrals at infinity vanish: the integrands $\hat{\boldsymbol{u}} \cdot \boldsymbol{\sigma}$ and $\boldsymbol{u} \cdot \hat{\boldsymbol{\sigma}}$ decay as $r^{-2} \ln r$ and $r^{-2}$, respectively, whereas $\mathrm{d} A=r \mathrm{~d} \theta$. On the body surface, the integrand $\hat{\boldsymbol{u}} \cdot \boldsymbol{\sigma}$ becomes simply $\hat{\boldsymbol{U}} \cdot \boldsymbol{\sigma}$, and this integral vanishes owing to the force-free condition. The only integral that survives is

$$
\int_{\Gamma} \boldsymbol{u} \cdot \hat{\boldsymbol{\sigma}} \cdot \hat{\boldsymbol{n}} \mathrm{d} A=\boldsymbol{U}_{0} \cdot \hat{\boldsymbol{F}}+\int \boldsymbol{u}_{s} \cdot \hat{\boldsymbol{\sigma}} \cdot \hat{\boldsymbol{n}} \mathrm{d} A
$$

which reduces to

$$
\boldsymbol{U}_{0}=-\frac{1}{2 \pi} \int \boldsymbol{u}_{s} \mathrm{~d} \theta
$$

for a circular cylinder, for which $\hat{\boldsymbol{\sigma}} \cdot \hat{\boldsymbol{n}}$ is constant.

We gratefully acknowledge support from the NSF Mathematical Sciences Postdoctoral Fellowship and Lee A. Dubridge Prize Postdoctoral Fellowship (T.M. S.) and the US Army through the Institute for Soldier Nanotechnologies, under Contract DAAD-19-02-0002 with the US Army Research Office (M.Z. B.).

\section{REFERENCES}

AJDARI, A. 1995 Electroosmosis on inhomogeneously charged surfaces. Phys. Rev. Lett. 75, 755-758.

AJDARI, A. 1996 Generation of transverse fluid currents and forces by an electric field: electroosmosis on charge-modulated and undulated surfaces. Phys. Rev. E 53, 4996-5005. 
AJdari, A. 2000 Pumping liquids using asymmetric electrode arrays. Phys. Rev. E 61, R45-R48.

AjDari, A. $2002 a$ Electrokinetic 'ratchet' pumps for microfluidics. Appl. Phys. A 75, 271-274.

AJDARI, A. $2002 b$ Transverse electrokinetic and microfluidic effects in micropatterned channels: lubrication analysis for slab geometries. Phys. Rev. E 6501, art. 016301.

Anderson, J. L. 1989 Colloid transport by interfacial forces. Annu. Rev. Fluid Mech. 21, 61-99.

Anker, J. N. \& Kopelman, R. 2003 Magnetically modulated optical nanoprobes. Appl. Phys. Lett. 82, $1102-1104$.

Bazant, M. Z. 2004 Conformal mapping of some non-harmonic functions in transport theory. Proc. R. Soc. Lond. A Math. Phys. Engng Sci. 460 (2045), 1433-1452.

BazANT, M. Z. \& SQuires, T. M. 2004 Induced-charge electrokinetic phenomena: theory and microfluidic applications. Phys. Rev. Lett. 92, art. 066101.

Bazant, M. Z., Thornton, K. \& Ajdari, A. 2004 Diffuse-charge dynamics in electrochemical systems. Phys. Rev. E 70, 021506.

Berg, H. C. 2003 The rotary motor of bacterial flagella. Annu. Rev. Biochem. 72, 19-54.

Brenner, H. 1964 The Stokes resistance of an arbitrary particle. 4. Arbitrary fields of flow. Chem. Engng Sci. 19, 703-727.

Brown, A. B. D., Smith, C. G. \& Rennie, A. R. 2001 Pumping of water with AC electric fields applied to asymmetric pairs of microelectrodes. Phys. Rev. E 63, art. 016305.

BRUIN, G. J. M. 2000 Recent developments in electrokinetically driven analysis on microfabricated devices. Electrophoresis 21, 3931-3951.

CHU, K. T. 2005 Asymptotic analysis of extreme electrochemical transport. PhD thesis, MIT.

Dukhin, S. S. 1993 Nonequilibrium electric surface phenomena. Adv. Colloid Interface Sci. 44, $1-134$.

Dukhin, S. S. \& Derjaguin, B. V. 1974 Electrokinetic phenomena. In Surface and Colloid Science, vol. 7. John Wiley.

Dukhin, S. S. \& Shilov, V. N. 1974 Dielectric Phenomena and the Double Layer in Disperse Systems and Polyelectrolytes. Wiley.

Dukhin, S. S. \& Shilov, V. N. 1980 Kinetic aspects of electrochemistry of disperse systems. 2. Induced dipole-moment and the nonequilibrium double-layer of a colloid particle. $A d v$. Colloid Interface Sci. 13, 153-195.

FAIR, M. C. \& Anderson, J. L. 1990 Electrophoresis of dumbbell-like colloidal particles. Intl J. Multiphase Flow 16, 663-679.

FAIR, M. C. \& ANDERSON, J. L. 1992 Electrophoresis of heterogeneous colloids - doublets of dissimilar particles. Langmuir 8, 2850-2854.

Finkel, N. H., LoU, X. H., WANG, C. Y. \& He, L. 2004 Barcoding the microworld. Anal. Chem. 76, 353A-359A.

Gamayunov, N. I. \& Murtsovkin, V. A. 1987 Motion of disperse particles in a uniform alternating electric-field. Colloid J. USSR 49, 543-544.

Gamayunov, N. I., Murtsovkin, V. A. \& Dukhin, A. S. 1986 Pair interaction of particles in electric-field. 1. Features of hydrodynamic interaction of polarized particles. Colloid J. USSR 48, 197-203.

Giddings, J. C. 1991 Unified Separation Science. John WIley.

Gitlin, I., Stroock, A. D., Whitesides, G. M. \& Ajdari, A. 2003 Pumping based on transverse electrokinetic effects. Appl. Phys. Lett. 83, 1486-1488.

Gonzalez, A., Ramos, A., Green, N. G., Castellanos, A. \& Morgan, H. 2000 Fluid flow induced by nonuniform AC electric fields in electrolytes on microelectrodes. II. A linear double-layer analysis. Phys. Rev. E 61, 4019-4028.

Green, N. G., Ramos, A., Gonzalez, A., Morgan, H. \& Castellanos, A. 2002 Fluid flow induced by nonuniform AC electric fields in electrolytes on microelectrodes. III. Observation of streamlines and numerical simulation. Phys. Rev. E 66, art. 026305.

Hinch, E. J. 1991 Perturbation Methods. Cambridge University Press.

JACKSON, J. D. 1975 Classical Electrodynamics. John Wiley.

JefFreY, D. J. \& ONishi, Y. 1981 The slow motion of a cylinder next to a plane wall. Q. J. Mech. Appl. Maths. 34, 129-137.

JoNES, T. B. \& WASHizU, M. 1996 Multipolar dielectrophoretic and electrorotation theory. J. Electrostat. 37, 121-134. 
Kinosita, K., Adachi, K. \& IтоH, H. 2004 Rotation of F-1 ATPase: how an ATP-driven molecular machine may work. Annu. Rev. Biophys. Biomolec. Struct. 33, 245-268.

Levich, V. G. 1962 Physicochemical Hydrodynamics. Prentice-Hall.

Levitan, J. A. 2005 Experimental investigation of induced-charge electro-osmosis. PhD thesis, MIT.

Levitan, J. A., Devasenathipathy, S., Studer, V., Thorsen, T., Squires, T. M. \& Bazant, M. Z. 2005 Experimental observation of induced-charge electro-osmosis around a metal wire in a microchannel. Coll. Surf. A 267, 122-132.

Lion, N., Rohner, T. C., Dayon, L., Arnaud, I. L., Damoc, E., Youhnovski, N., Wu, Z. Y., Roussel, C., Josserand, J., Jensen, H., Rossier, J. S., Przybylski, M. \& Girault, H. H. 2003 Microfluidic systems in proteomics. Electrophoresis 24, 3533-3562.

Long, D. \& AJdARI, A. 1996 Electrophoretic mobility of composite objects in free solution: application to DNA separation. Electrophoresis 17, 1161-1166.

Long, D. \& AJDARI, A. 1998 Symmetry properties of the electrophoretic motion of patterned colloidal particles. Phys. Rev. Lett. 81, 1529-1532.

Lyklema, J. 1995 Fundamentals of Interface and Colloid Science, vol. 2. Academic.

Morgan, H. \& GreEn, N. G. 2003 AC Electrokinetics: Colloids and Nanoparticles. Research Studies Press, Philadelphia.

Mpholo, M., Smith, C. G. \& Brown, A. B. D. 2003 Low voltage plug flow pumping using anisotropic electrode arrays. Sens. Act. B 92, 262-268.

Murtsovkin, V. A. 1996 Nonlinear flows near polarized disperse particles. Colloid J. 58, 341-349.

Murtsovkin, V. A. \& Mantrov, G. I. 1990 Study of the motion of anisometric particles in a uniform variable electric-field. Colloid J. USSR 52, 933-936.

Newman, J. S. 1991 Electrochemical Systems. Prentice-Hall.

Nicewarner-Pena, S. R., Freeman, R. G., Reiss, B. D., He, L., Pena, D. J., Walton, I. D., Cromer, R., Keating, C. D. \& Natan, M. J. 2001 Submicrometer metallic barcodes. Science 294, $137-141$.

Oleson, L. H., Bruus, H. \& AJdARI, A. 2006 AC electrokinetic micropumps: the effect of geometrical confinement, Faradaic current injection, and nonlinear surface capacitance. Phys. Rev. E in press.

PoHL, H. A. 1978 Dielectrophoresis: the behaviour of neutral matter in nonuniform electric fields. Cambridge University Press.

Proudman, I. \& Pearson, J. R. A. 1957 Expansions at small Reynolds numbers for the flow past a sphere and a circular cylinder. J. Fluid Mech. 2, 237-262.

Ramos, A., Morgan, H., Green, N. G. \& Castellanos, A. 1998 AC electrokinetics: a review of forces in microelectrode structures. J. Phys. D 31, 2338-2353.

Ramos, A., Morgan, H., Green, N. G. \& Castellanos, A. 1999 AC electric-field-induced fluid flow in microelectrodes. J. Colloid Interface Sci. 217, 420-422.

Ramos, A., Gonzalez, A., Castellanos, A., Green, N. G. \& Morgan, H. 2003 Pumping of liquids with AC voltages applied to asymmetric pairs of microelectrodes. Phys. Rev. E 67, art. 056302.

Reyes, D. R., Iossifidis, D., Auroux, P. A. \& Manz, A. 2002 Micro total analysis systems. 1. Introduction, theory, and technology. Anal. Chem. 74, 2623-2636.

Rose, K. \& Santiago, J. G. 2006 Rotational electrophoresis of striped metallic microrods. Phys. Rev. E submitted.

Russel, W. B., Saville, D. A. \& Schowalter, W. R. 1989 Colloidal Dispersions. Cambridge University Press.

Saintillan, D., Darve, E. \& Shaqfeh, E. S. G. 2006 Hydrodynamic interactions in the inducedcharge electrophoresis of colloidal rod dispersions. J. Fluid Mech. (in press).

Shilov, V. N. \& Éstrela-Lópis, V. R. 1975 Theory of movement of spherical particles of a suspension in an inhomogeneous electric field. In Research in Surface Forces, Vol. 4: Surface Forces in Thin Films and Disperse Systems, p. 121. Consultants Bureau, New York.

Shilov, V. N. \& Simonova, T. S. 1981 Polarization of electric double-layer of disperse particles and dipolophoresis in a steady (DC) field. Colloid J. USSR 43, 90-96.

Simonov, I. N. \& Shilov, V. N. 1977 Theory of low-frequency dielectric-dispersion of a suspension of ideally polarizable spherical-particles. Colloid J. USSR 39, 775-780.

Simonova, T. S., Shilov, V. N. \& Shramko, O. A. 2001 Low-frequency dielectrophoresis and the polarization interaction of uncharged spherical particles with an induced Debye atmosphere of arbitrary thickness. Colloid J. 63, 108-115. 
Slater, G. W., Kenward, M., McCormick, L. C. \& Gauthier, M. G. 2003 The theory of DNA separation by capillary electrophoresis. Curr. Opin. Biotech. 14, 58-64.

Squires, T. M. 2001 Effective pseudo-potentials of hydrodynamic origin. J. Fluid Mech. 443, 403-412.

Squires, T. M. \& BazAnt, M. Z. 2004 Induced-charge electro-osmosis. J. Fluid Mech. 509, 217-252.

Squires, T. M. \& Quake, S. R. 2005 Microfluidics: fluid physics at the nanoliter scale. Rev. Mod. Phys. 77, 977-1026.

Stone, H. A. \& Samuel, A. D. T. 1996 Propulsion of microorganisms by surface distortions. Phys. Rev. Lett. 77, 4102-4104.

Stone, H. A., Stroock, A. D. \& AJdari, A. 2004 Engineering flows in small devices: microfluidics toward a lab-on-a-chip. Annu. Rev. Fluid Mech. 36, 381411.

Studer, V., Pepin, A., Chen, Y. \& Ajdari, A. 2002 Fabrication of microfluidic devices for AC electrokinetic fluid pumping. Microelectron. Engng 61-2, 915-920.

Studer, V., Pepin, A., Chen, Y. \& Ajdari, A. 2004 An integrated AC electrokinetic pump in a microfluidic loop for fast and tunable flow control. Analyst 129, 944-949.

Tegenfeldt, J. O., Prinz, C., Cao, H., Huang, R. L., Austin, R. H., Chou, S. Y., Cox, E. C. \& Sturm, J. C. 2004 Micro- and nanofluidics for DNA analysis. Anal. Bioanal. Chem. 378, $1678-1692$.

Ugaz, V. M., Elms, R. D., Lo, R. C., Shaikh, F. A. \& Burns, M. A. 2004 Microfabricated electrophoresis systems for DNA sequencing and genotyping applications: current technology and future directions. Phil. Trans. R. Soc. Lond. A 362, 1105-1129.

Verpoorte, E. 2002 Microfluidic chips for clinical and forensic analysis. Electrophoresis 23, 677-712.

VIovy, J. L. 2000 Electrophoresis of DNA and other polyelectrolytes: physical mechanisms. Rev. Mod. Phys. 72, 813-872.

WANG, X. J., Wang, X. B. \& Gascoyne, P. R. C. 1997 General expressions for dielectrophoretic force and electrorotational torque derived using the maxwell stress tensor method. J. Electrost. 39, $277-295$.

Washizu, M. \& Jones, T. B. 1994 Multipolar dielectrophoretic force calculation. J. Electrost. 33, $187-198$.

YARIV, E. 2005 Induced-charge electrophoresis of nonspherical particles. Phys. Fluids 17, 051702. 